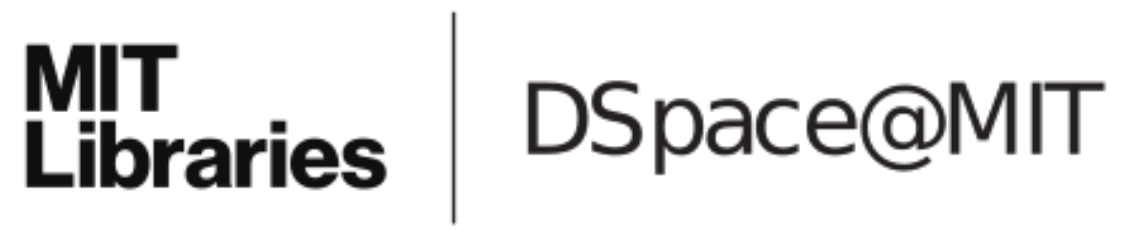

\author{
MIT Open Access Articles
}

Fluctuating surface currents: An algorithm for efficient prediction of Casimir interactions among arbitrary materials in arbitrary geometries

The MIT Faculty has made this article openly available. Please share how this access benefits you. Your story matters.

Citation: Reid, M. T. Homer, Jacob White, and Steven G. Johnson. “Fluctuating surface currents: An algorithm for efficient prediction of Casimir interactions among arbitrary materials in arbitrary geometries." Physical Review A 88, no. 2 (August 2013). (C 2013 American Physical Society

As Published: http://dx.doi.org/10.1103/PhysRevA.88.022514

Publisher: American Physical Society

Persistent URL: http://hdl.handle.net/1721.1/81397

Version: Final published version: final published article, as it appeared in a journal, conference proceedings, or other formally published context

Terms of Use: Article is made available in accordance with the publisher's policy and may be subject to US copyright law. Please refer to the publisher's site for terms of use. 


\title{
Fluctuating surface currents: An algorithm for efficient prediction of Casimir interactions among arbitrary materials in arbitrary geometries
}

\author{
M. T. Homer Reid, ${ }^{1, *}$ Jacob White, ${ }^{1,2}$ and Steven G. Johnson ${ }^{1,3}$ \\ ${ }^{1}$ Research Laboratory of Electronics, Massachusetts Institute of Technology, Cambridge, Massachusetts 02139, USA \\ ${ }^{2}$ Department of Electrical Engineering and Computer Science, Massachusetts Institute of Technology, Cambridge, Massachusetts 02139, USA \\ ${ }^{3}$ Department of Mathematics, Massachusetts Institute of Technology, Cambridge, Massachusetts 02139, USA
}

(Received 29 February 2012; revised manuscript received 23 May 2013; published 23 August 2013)

\begin{abstract}
This paper presents a method for the efficient numerical computation of Casimir interactions between objects of arbitrary geometries, composed of materials with arbitrary frequency-dependent electrical properties. Our method formulates the Casimir effect as an interaction between effective electric and magnetic current distributions on the surfaces of material bodies and obtains Casimir energies, forces, and torques from the spectral properties of a matrix that quantifies the interactions of these surface currents. The method can be formulated and understood in two distinct ways: (1) as a consequence of the familiar stress-tensor approach to Casimir physics, or, alternatively, (2) as a particular case of the path-integral approach to Casimir physics, and we present both formulations in full detail. In addition to providing an algorithm for computing Casimir interactions in geometries that could not be efficiently handled by any other method, the framework proposed here thus achieves an explicit unification of two seemingly disparate approaches to computational Casimir physics.
\end{abstract}

DOI: 10.1103/PhysRevA.88.022514

PACS number(s): 31.30.jh, 03.70.+k, 42.50.Lc, 03.65.Db

\section{INTRODUCTION}

This paper presents a method for the efficient numerical computation of Casimir interactions between objects of arbitrary geometries, composed of materials with arbitrary frequency-dependent electrical properties. Our method formulates the Casimir effect as an interaction between effective electric and magnetic currents on the surfaces of material bodies and obtains Casimir energies, forces, and torques from the spectral properties of a matrix that quantifies the interactions of these surface currents. Our final formulas for Casimir quantities-Eqs. (I) below-may be derived in two distinct ways: (a) by integrating the Maxwell stress tensor over a closed bounding surface, as is commonly done in purely numerical approaches to Casimir computation [1], but with the distinction that here we evaluate the surface integral analytically, or (b) by evaluating a path-integral expression for the Casimir energy, as is commonly done in quasianalytical approaches to Casimir physics [2], but with the distinction that here we are not restricted to the use of geometryspecific special functions. In this paper, we present these two distinct derivations of our master formulas (1) and compare our approach to existing computational Casimir methods. A free, open-source software package implementing our method is available [3]; the technical details of this and other numerical implementations of our method will be discussed elsewhere.

Results obtained using our technique have appeared in print before [4-8], and Refs. [4,5] briefly sketched the path-integral derivation of our method, but omitted many details. The purposes of the present paper are to furnish a complete presentation of the path-integral derivation and to present the alternative stress-tensor derivation. By arriving at identical formulas—our master formulas, Eq. (1)—from the

\footnotetext{
*homereid@mit.edu; http://www.mit.edu/ homereid
}

two seemingly disparate starting points of path integrals and stress tensors, we explicitly demonstrate the equivalence of these two formulations of Casimir physics.

In particular, our demonstration of this equivalence furnishes an alternative demonstration that the Maxwell stress tensor in dispersive media-questionable under ordinary circumstances-is, in fact, valid in the thermodynamic context, as has been argued on other grounds by Pitaevskii [9] and by Philbin $[10,11]$ in the context of the canonical quantization of macroscopic electromagnetism. An algebraic equivalence similar to ours, but relating a path-integral expression to the energy density instead of the Maxwell stress tensor, was demonstrated in Ref. [12], which used this equivalence to explain why the dispersive energy density (which is valid in ordinary electrodynamics only for negligible dissipation [13]) is the appropriate quantity to consider in the context of thermal and quantum fluctuations. Our work does for the stress tensor what Ref. [12] does for the energy density. (An alternative approach to relating the stress-tensor picture to the energy viewpoint was suggested in Ref. [14], but details were omitted; also, the method was restricted to geometries that admit a separating plane between objects, whereas the method of this paper has no such restriction and is applicable even to geometries containing objects with interpenetrating features.)

Although Casimir physics has been with us for some 7 decades [15], the past 15 years have witnessed a renaissance of interest in the field, driven by laboratory observations of Casimir phenomena in an increasingly complex variety of geometric and material configurations [16-20]. Whereas the theoretical methods used in the original Casimir prediction [15] were restricted to the case of simple geometries and idealized materials, recent experimental progress has spurred the development of theoretical techniques for predicting Casimir forces among bodies of arbitrary shapes and material properties. Such general-purpose Casimir methods have typically pursued one of two general strategies. 
A first approach [2,21-26] seeks to exploit geometrical symmetries by approximating Casimir quantities as expansions in special functions (solutions of the scalar or vector Helmholtz equation in various coordinate systems) that encode global geometric information in a concise way. (Techniques of this sort are often known as spectral methods [27].) These methods have the virtue of yielding compact expressions relating Casimir energies, forces, and torques to linear-algebra operations (matrix inverse, determinant, and trace) on matrices describing the interactions of the global basis functions. As is commonly true for spectral methods, the expressions are rapidly convergent (in the sense of obtaining accurate numerical results with low-dimensional truncations of the matrices) for highly symmmetric geometries, but less wellsuited to asymmetric configurations, where the very geometric specificity encoded in the closed-form Helmholtz solutions becomes more curse than blessing and requires the specialfunction expansions to be carried out to high orders for even moderate numerical precision.

An alternative approach is a numerical implementation of the stress-tensor formulation of Casimir physics pioneered by Lifshitz et al. [28,29]. Here the Casimir force on a body is obtained by integrating the Maxwell stress tensorsuitably averaged over thermal and quantum-mechanical fluctuations-over a fictitious bounding surface surrounding the body; in modern numerical approaches [1,30-32] the integral is evaluated by numerical cubature (that is, as a weighted sum of integrand samples) with values of the stress tensor at each cubature point computed by solving numerical electromagnetic scattering problems. As compared to the special-function approach, this technique has the virtue of great generality, as it allows one to take advantage of the wide range of existing numerical techniques for solving scattering problems involving arbitrarily complex geometries and materials. The drawback is that the spatial integral over the bounding surface adds a layer of conceptual and computational complexity that is absent from the special-function approach.

In this paper we show how the best features of these two approaches may be combined to yield a technique for Casimir computations. Our fluctuating-surface-current (FSC) approach expresses Casimir energies, forces, and torques among bodies of arbitrary geometries and material properties in terms of interactions among effective electric and magnetic currents flowing on the object surfaces. The method borrows techniques from surface-integral-equation formulations of electromagnetic scattering [33] to represent objects entirely in terms of their surfaces-thus retaining the full flexibility of the numerical stress-tensor method in handling arbitrarily complex asymmetric geometries-but bypasses the unwieldy numerical cubatures of the usual stress-tensor approach to obtain Casimir energies, forces, and torques directly from linear-algebra operations (matrix inverse, determinant, and trace) on matrices describing the interactions of the surface currents, thus retaining the conceptual simplicity and computational ease of the usual special-function approach.

The FSC formulas for the zero-temperature Casimir energy, force, and torque are

$$
\mathcal{E}=\frac{\hbar}{2 \pi} \int_{0}^{\infty} d \xi \ln \frac{\operatorname{det} \mathbf{M}(\xi)}{\operatorname{det} \mathbf{M}_{\infty}(\xi)}
$$

$$
\begin{aligned}
& \mathcal{F}_{i}=-\frac{\hbar}{2 \pi} \int_{0}^{\infty} d \xi \operatorname{Tr}\left\{\mathbf{M}^{-1}(\xi) \cdot \frac{\partial \mathbf{M}(\xi)}{\partial \mathbf{r}_{i}}\right\} \\
& \mathcal{T}=-\frac{\hbar}{2 \pi} \int_{0}^{\infty} d \xi \operatorname{Tr}\left\{\mathbf{M}^{-1}(\xi) \cdot \frac{\partial \mathbf{M}(\xi)}{\partial \theta}\right\}
\end{aligned}
$$

where $\mathbf{M}(\xi)$ is just the usual matrix that arises in the boundary-element method of electromagnetic scattering, but here evaluated at a purely imaginary angular frequency $\omega=$ $i \xi$. [Boundary-element methods and the precise form of $\mathbf{M}(\xi)$ are reviewed in Appendix B.] In (I), the $\xi$ integrals extend over the positive imaginary frequency axis, and the partial derivative in the force (torque) equation is taken with respect to a rigid displacement (rotation) of the object in question. Readers familiar with scattering-matrix methods for Casimir computations [2,23-26] will note the striking similarity of our Eq. (1a) to the Casimir energy formulas reported in those works (such as Eq. 5.13 of Ref. [2]); in both cases, the Casimir energy is obtained by integrating over the imaginary frequency axis, with an integrand expressed as a ratio of matrix determinants. The difference lies in the meaning of the matrices in the two cases; whereas the matrix in typical scattering-matrix Casimir methods describes the interactions of incoming and outgoing wave solutions of Maxwell's equations, the matrix in our Eqs. (1) describes the interactions of surface currents flowing on the boundaries of the interacting objects in a Casimir geometry. This distinction has important ramifications for the convenience and generality of our method.

In traditional scattering-matrix Casimir methods, the matrix that enters equations like (1) describes interactions among the elements of a basis of known solutions of Maxwell's equations propagating to and from the interacting bodies. Such treatments afford a highly efficient description of scattering in the handful of geometries for which analytical solutions are available - such as incoming and outgoing spherical waves for spherical scatterers, left- and right-traveling plane waves for planar geometries, cylindrical wave for cylinders, etc.-but may be particularly inefficient for describing more general objects, as, for example, if one attempts to describe scattering from a cube using a basis of spherical waves. Moreover, practical implementations of these methods require significant retooling to accommodate shapes of objects; if, for example, having formulated the method for spheres, one wishes instead to treat spheroids, one must recompute Maxwell solutions in a coordinate system and reformulate the matrices in equations like (1) to describe the interactions of these solutions.

In contrast, the matrix in our Eqs. (1) describes the interactions of surface currents flowing on the surfaces of the interacting objects in a Casimir geometry, as discussed in detail in Appendix B. A crucial advantage of this description is that the basis we use to represent surface currents is arbitrary. The basis functions are not required to solve the wave equation or any other equation, and the choice of basis is thus liberated from the underlying physics of the problem; we are free to choose a basis that efficiently represents any given geometry. One convenient choice-though by no means the only possibility - is a basis of localized functions conforming to a nonuniform surface-mesh discretization (Fig. 5), where the mesh may be automatically generated for arbitrarily complex geometries [34]. A particular advantage of this type of basis 
is that, once we have implemented our method using basis functions of this type, we can apply it to arbitrary geometries with almost no additional effort; in particular, having applied the method to spheres, it is essentially effortless to apply it to cubes (Sec. V).

The objective of this paper is to provide two separate derivations of the master formulas (1), one based on the stress-tensor formalism and making no reference to path integration, and a second based on path integrals and making no reference to stress tensors. These derivations contain a number of theoretical innovations beyond the practical usefulness of the method itself; in particular, in the stress-tensor derivation we state and prove a new integral identity involving the homogeneous dyadic Green's functions of Maxwell's equations (Appendix C), while in the path-integral derivation we introduce a surface-current representation of the Lagrange multipliers that constrain functional integrations over the electromagnetic field, which we expect to be a tool of general use in quantum field theory.

The Casimir method described in this paper is closely related to the surface-integral-equation (SIE) formulation of classical electromagnetic theory. Although well known in the engineering literature [33], this technique has not been extensively discussed in the Casimir literature, and for this reason we provide in Appendix $B$ a brief review of SIE theory. The majority of this Appendix is a review of standard material, with the exception of the explicit expressions for the dyadic Green's functions that we derive in Appendix B3. In Secs. II and III, which constitute the centerpiece of the paper, we present two separate derivations of the master FSC formulas (1); one derivation starts from the stress-tensor approach to Casimir physics (Sec. II), while an independent derivation starts from a path-integral expression for the Casimir energy (Sec. III). In Sec. IV, we note an important practical simplification that follows from the structure of the matrices in Eqs. (1). In Sec. V we validate our method by using it to reproduce known results, then illustrate its generality by applying it to geometries that would be difficult to address using existing Casimir methods. (Further examples of the utility of our method may be found in Refs. [4-8].) Our conclusions are presented in Sec. VI. A number of technical details are discussed in the appendixes. Technical details of practical numerical implementations, as well as additional computational applications, will be discussed elsewhere.

\section{STRESS-TENSOR DERIVATION OF THE FSC CASIMIR FORMULAS}

The stress-tensor approach to Casimir physics relates Casimir forces to classical dyadic Green's functions (DGFs). This technique was pioneered by Dzyaloshinskii, Lifshitz, and Pitaevskii (DLP) in the 1950s [28,29] and has remained an important computational strategy ever since [35,36]; in particular, modern numerical algorithms for computing Casimir forces between bodies of complex geometries have tended to use the stress-tensor approach, with values for the relevant DGFs computed numerically [1,30-32]. Here, after briefly reviewing the formalism relating Casimir forces to DGFs (Sec. II A), we show that the concise SIE expressions for the DGFs that we derive in Appendix B (Appendix B3) afford a significant simplification of the usual computational procedure. In particular, we show that the surface integral of the stress tensor, which in previous work has typically been evaluated by numerical cubature, may, in fact, be evaluated analytically for an arbitrary closed surface of integration, leaving behind a simple expression relating the Casimir force to the trace of a certain matrix.

\section{A. A review of stress-tensor Casimir physics}

In the stress-tensor approach, the $i$-directed Casimir force on a body is obtained by integrating the expectation value of the Maxwell stress tensor over a closed bounding surface surrounding the body:

$$
\begin{gathered}
\mathcal{F}_{i}=\int_{0}^{\infty} \frac{d \xi}{\pi} F_{i}(\xi), \\
F_{i}(\xi)=\oint_{\mathcal{C}}\left\langle T_{i j}(\xi ; \mathbf{x})\right\rangle \hat{\mathbf{n}}_{j}(\mathbf{x}) d \mathbf{x} .
\end{gathered}
$$

Here the integration surface $\mathcal{C}$ may be the surface of the body in question or any fictitious closed surface in space bounding the body (as in Fig. 4), and the expectation value is taken with respect to quantum and thermal fluctuations. The expectation value of $T_{i j}$ is next written in terms of the components of the electric and magnetic fields,

$$
\begin{aligned}
\left\langle T_{i j}\right\rangle= & \epsilon\left\langle E_{i} E_{j}\right\rangle+\mu\left\langle H_{i} H_{j}\right\rangle \\
& -\frac{\delta_{i j}}{2}\left[\epsilon\left\langle E_{k} E_{k}\right\rangle+\mu\left\langle H_{k} H_{k}\right\rangle\right] .
\end{aligned}
$$

[Here it is understood that $\epsilon=\epsilon_{0} \epsilon^{e}$ and $\mu=\mu_{0} \mu^{e}$ are the (spatially constant) permittivity and permeability of the exterior medium at the frequency in question; $\epsilon^{e}, \mu^{e}$ are the dimensionless relative quantities.] Finally, the fluctuationdissipation theorem is invoked to relate the expectation values of products of field components to scattering DGFs [28,29]; at temperature $T=0$, the relations read

$$
\begin{aligned}
\left\langle E_{i}(\xi, \mathbf{x}) E_{j}\left(\xi, \mathbf{x}^{\prime}\right)\right\rangle & =-\hbar \xi \mathcal{G}_{i j}^{\mathrm{EE}}\left(\xi ; \mathbf{x}, \mathbf{x}^{\prime}\right), \\
\left\langle H_{i}(\xi, \mathbf{x}) H_{j}\left(\xi, \mathbf{x}^{\prime}\right)\right\rangle & =-\hbar \xi \mathcal{G}_{i j}^{\mathrm{MM}}\left(\xi ; \mathbf{x}, \mathbf{x}^{\prime}\right),
\end{aligned}
$$

where, as discussed in Appendix $\mathrm{A}, \mathcal{G}^{\mathrm{EE}}\left(\xi, \mathbf{x}, \mathbf{x}^{\prime}\right)$ is the scattered portion of the electric field at $\mathbf{x}$ due to an electric current source at $\mathbf{x}^{\prime}$, all quantities having time dependence $\propto e^{+\xi t}$; similarly, $\mathcal{G}^{\mathrm{MM}}$ gives the scattered magnetic field due to a magnetic current source. (In the original work, DLP wrote $\nabla \times \nabla \times \mathcal{G}^{\mathrm{EE}}$ in place of $\mathcal{G}^{\mathrm{MM}}$; the equivalence of the two quantities has been discussed, e.g., in Ref. [1].)

Inserting (4) and (5) into (3) yields an expression for the Casimir force-per-unit-frequency in terms of scattering DGFs:

$$
\begin{aligned}
F_{i}(\xi)= & -\frac{\hbar \xi}{\pi} \oint_{\mathcal{C}}\left\{\epsilon \mathcal{G}_{i j}^{\mathrm{EE}}+\mu \mathcal{G}_{i j}^{\mathrm{MM}}\right. \\
& \left.-\frac{\delta_{i j}}{2}\left[\epsilon \mathcal{G}_{k k}^{\mathrm{EE}}+\mu \mathcal{G}_{k k}^{\mathrm{MM}}\right]\right\} \hat{\mathbf{n}}_{j} d \mathbf{x} .
\end{aligned}
$$

Equation (6) is the starting point of many numerical Casimir studies, as it reduces the computation of Casimir forces to the computational of classical DGFs. In principle, the DGFs in question may be computed using any of the myriad available numerical techniques for classical scattering problems; to 
date, numerical Casimir investigations using both the finitedifference method [30,31] and the discretized SIE method reviewed in Appendix B [32,37] have appeared. In these studies, the surface integral in (6) is evaluated by numerical cubature, with the values of the integrand at each cubature point computed by solving numerical scattering problems.

Here we proceed in a different direction. Instead of taking Eq. (6) as the jumping-off point for a numerical investigation, we continue the analytical development one step further by inserting our explicit SIE expressions (B19) and (B22) into Eq. (6) and analyzing the result. As we will see, this step will allow us to evaluate the surface integral in (6) analytically, eliminating the need for numerical cubature and resulting in a compact matrix-trace formula for the Casimir force.

Because the thrust of the argument is easiest to present in the simplest case of perfectly electrically conducting (PEC) bodies, we begin with that case in Sec. II B, leaving the treatment of general materials to Sec. II C.

\section{B. Stress-tensor derivation of FSC formulas for PEC objects}

In Sec. B 3 we derive explicit SIE expressions for the DGFs that enter into the integrand of (6); for the case of PEC scatterers, the relevant expressions are Eqs. (B19). Our strategy here will be to insert these expressions into (6) and analyze the result; to facilitate this procedure, it is convenient first to write Eqs. (B19) in a slightly different form by (a) expressing the four $\boldsymbol{\Gamma}$ dyadics in terms of the two $\mathbf{G}$ and $\mathbf{C}$ dyadics (Appendix A) and (b) writing out inner products like $\langle\mathbf{f} \mid \boldsymbol{\Gamma}\rangle$ explicitly as integrals over the supports of the basis function $\mathbf{f}$ [compare Eqs. (B5), (B6), and (B7)]. Then the quantities that enter into the integrand of (6) are

$$
\begin{aligned}
\epsilon \mathcal{G}_{i j}^{\mathrm{EE}}(\mathbf{x}, \mathbf{x}) & =-\epsilon \sum_{\alpha \beta}\left\langle\boldsymbol{\Gamma}_{i}^{\mathrm{EE}, e}(\mathbf{x}) \mid \mathbf{f}_{\alpha}\right\rangle W_{\alpha \beta}\left\langle\mathbf{f}_{\beta} \mid \boldsymbol{\Gamma}_{j}^{\mathrm{EE}, e}\left(\mathbf{x}^{\prime}\right)\right\rangle \\
& =-\mu_{0} \mu^{e}\left(\kappa^{e}\right)^{2} \sum_{\alpha \beta} W_{\alpha \beta}\left\{\int_{\sup _{\alpha}} G_{i k}\left(\mathbf{r}_{\alpha}, \mathbf{x}\right) f_{\alpha k}\left(\mathbf{r}_{\alpha}\right) d \mathbf{r}_{\alpha}\right\}\left\{\int_{\sup _{\beta}} f_{\beta \ell}\left(\mathbf{r}_{\beta}\right) G_{\ell j}\left(\mathbf{x}, \mathbf{r}_{\beta}\right) d \mathbf{r}_{\beta}\right\}, \\
\mu \mathcal{G}_{i j}^{\mathrm{MM}}(\mathbf{x}, \mathbf{x}) & =-\mu \sum_{\alpha \beta}\left\langle\Gamma_{i}^{\mathrm{ME}, e}(\mathbf{x}) \mid \mathbf{f}_{\alpha}\right\rangle W_{\alpha \beta}\left\langle\mathbf{f}_{\beta} \mid \boldsymbol{\Gamma}_{j}^{\mathrm{EM}, e}\left(\mathbf{x}^{\prime}\right)\right\rangle \\
& =+\mu_{0} \mu^{e}\left(\kappa^{e}\right)^{2} \sum_{\alpha \beta} W_{\alpha \beta}\left\{\int_{\sup _{\alpha}} C_{i k}\left(\mathbf{r}_{\alpha}, \mathbf{x}\right) f_{\alpha k}\left(\mathbf{r}_{\alpha}\right) d \mathbf{r}_{\alpha}\right\}\left\{\int_{\sup \mathbf{f}_{\beta}} f_{\beta \ell}\left(\mathbf{r}_{\beta}\right) C_{\ell j}\left(\mathbf{x}, \mathbf{r}_{\beta}\right) d \mathbf{r}_{\beta}\right\} .
\end{aligned}
$$

(Here $\kappa^{e}=\sqrt{\epsilon^{e} \mu^{e}} \xi$ is the imaginary wave number of the exterior medium, and we have suppressed the dependence of the $\mathbf{G}$ and $\mathbf{C}$ tensors on $\kappa^{e}$.) Note that both of these expressions have the same form: a sum over basis functions $\mathbf{f}_{\alpha}$ and $\mathbf{f}_{\beta}$, with a summand involving integrations over the supports of the basis functions. Indeed, Eqs. (7a) and (7b) are identical up to the different kernel functions ( $\mathbf{G}$ or $\mathbf{C}$ ) that enter into the integrals over basis functions. Note also that the variable $\mathbf{x}$, which is the integration variable in the surface integral in (6), appears in (7) only through these kernel functions. This implies that, after inserting (7) into (6), we will again have a sum of terms of this same form-a sum over basis functions, with the summand involving integrals over the basis functions - and, moreover, that many of the factors in this summand will be independent of the integration variable $\mathbf{x}$ in (6) and may thus be pulled outside the surface integral, which will now contain only factors of $\mathbf{G}$ and $\mathbf{C}$. The result is $\left(Z_{0}=\sqrt{\mu_{0} / \epsilon_{0}}, Z^{e}=\sqrt{\mu^{e} / \epsilon^{e}}\right)$,

$$
F_{i}(\xi)=\frac{\hbar}{\pi} \sum_{\alpha \beta} W_{\alpha \beta} Z_{0} Z^{e} \kappa^{e} \int_{\sup _{\alpha}} d \mathbf{r}_{\alpha} \int_{\sup _{\beta}} d \mathbf{r}_{\beta}\left\{f_{\alpha k}\left(\mathbf{r}_{\alpha}\right) \mathcal{I}_{i k \ell}\left(\mathbf{r}_{\alpha}, \mathbf{r}_{\beta}\right) f_{\beta \ell}\left(\mathbf{r}_{\beta}\right)\right\},
$$

where, as anticipated, the surface integral is now contained inside the definition of the $\mathcal{I}$ kernel:

$$
\mathcal{I}_{i k \ell}\left(\mathbf{r}_{\alpha}, \mathbf{r}_{\beta}\right) \equiv\left(\kappa^{e}\right)^{2} \oint_{\mathcal{C}}\left\{G_{i k}\left(\mathbf{r}_{\alpha}, \mathbf{x}\right) G_{\ell j}\left(\mathbf{x}, \mathbf{r}_{\beta}\right)-C_{i k}\left(\mathbf{r}_{\alpha}, \mathbf{x}\right) C_{\ell j}\left(\mathbf{x}, \mathbf{r}_{\beta}\right)-\frac{\delta_{i j}}{2}\left[G_{m k}\left(\mathbf{r}_{\alpha}, \mathbf{x}\right) G_{\ell m}\left(\mathbf{x}, \mathbf{r}_{\beta}\right)-C_{m k}\left(\mathbf{r}_{\alpha}, \mathbf{x}\right) C_{\ell m}\left(\mathbf{x}, \mathbf{r}_{\beta}\right)\right]\right\} \hat{\mathbf{n}}_{j} d \mathbf{x}
$$

The fact that $\mathbf{W}$ is a symmetric matrix $\left(W_{\alpha \beta}=W_{\beta \alpha}\right)$ allows us to rewrite Eq. (8) to read

$$
F_{i}(\xi)=\frac{\hbar}{2 \pi} \sum_{\alpha \beta} W_{\alpha \beta} Z_{0} Z^{e} \kappa^{e} \int_{\sup \mathbf{f}_{\alpha}} d \mathbf{r}_{\alpha} \int_{\sup _{\beta}} d \mathbf{r}_{\beta}\left\{f_{\alpha k}\left(\mathbf{r}_{\alpha}\right) \overline{\mathcal{I}}_{i k \ell}\left(\mathbf{r}_{\alpha}, \mathbf{r}_{\beta}\right) f_{\beta \ell}\left(\mathbf{r}_{\beta}\right)\right\},
$$

where we have defined a symmetrized version of the $\mathcal{I}$ kernel:

$$
\overline{\mathcal{I}}_{i k \ell}\left(\mathbf{r}, \mathbf{r}^{\prime}\right) \equiv \mathcal{I}_{i k \ell}\left(\mathbf{r}, \mathbf{r}^{\prime}\right)+\mathcal{I}_{i \ell k}\left(\mathbf{r}^{\prime}, \mathbf{r}\right) .
$$

The point of this step is that, as demonstrated in Appendix $\mathrm{C}$, the surface integral in the definition of the $\overline{\mathcal{I}}$ kernel may be evaluated in closed form, for any topological two-sphere $\mathcal{C}$, with the result

$$
\overline{\mathcal{I}}_{i k \ell}\left(\mathbf{r}, \mathbf{r}^{\prime}\right)= \begin{cases}0, & \text { if } \quad \mathbf{r}, \mathbf{r}^{\prime} \text { lie both inside or both outside } \mathcal{C}, \\ \frac{\partial}{\partial \mathbf{r}_{i}^{\mathrm{I}}} G_{k \ell}\left(\mathbf{r}^{\mathrm{I}}, \mathbf{r}^{\mathrm{E}}\right), & \text { if } \quad \mathbf{r}, \mathbf{r}^{\prime} \text { lie on opposite sides of } \mathcal{C},\end{cases}
$$

where, in the second case, $\mathbf{r}^{\mathrm{I}}\left(\mathbf{r}^{\mathrm{E}}\right)$ is whichever of $\mathbf{r}, \mathbf{r}^{\prime}$ lies in the interior (exterior) of $\mathcal{C}$. 
Armed with the dichotomy (10), we can now analyze the quantity in curly brackets in (9). Recall that the bounding contour $\mathcal{C}$ encloses one of the objects in our Casimir geometry; call this object $\mathcal{O}_{1}$ and the remaining objects $\mathcal{O}_{2,3, \ldots}$. Equation (10) then tells us that the curly bracketed term in (9) vanishes except when precisely one of the basis functions $\left\{\mathbf{f}_{\alpha}, \mathbf{f}_{\beta}\right\}$ lies on the surface of object $\mathcal{O}_{1}$. When this condition is satisfied, the integral over basis functions in (9) reads

$$
\begin{aligned}
-Z_{0} Z^{e} \kappa^{e} \int_{\sup _{\alpha}} d \mathbf{r}_{\alpha} \int_{\sup \mathbf{f}_{\beta}} \\
\times d \mathbf{r}_{\beta}\left\{f_{\alpha k}\left(\mathbf{r}_{\alpha}\right)\left[\frac{\partial}{\partial \mathbf{r}_{\alpha i}} G_{k \ell}\left(\mathbf{r}_{\alpha}, \mathbf{r}_{\beta}\right)\right] f_{\beta \ell}\left(\mathbf{r}_{\beta}\right)\right\} \\
=\left\langle\mathbf{f}_{\alpha}\left|\frac{\partial}{\partial \mathbf{r}_{\alpha i}} \Gamma^{\mathrm{EE}, e}\right| \mathbf{f}_{\beta}\right\rangle .
\end{aligned}
$$

However, this is nothing but the derivative of the $\alpha, \beta$ element of the SIE matrix (B6) with respect to a rigid infinitesimal displacement of object $\mathcal{O}_{1}$ in the $i$ direction,

$$
=\frac{\partial}{\partial \mathbf{r}_{i}} M_{\alpha \beta} \text {. }
$$

Inserting this into (9), we find that the imaginary-frequency- $\xi$ contribution to the Casimir force is given simply by

$$
\begin{aligned}
F_{i}(\xi) & =\frac{\hbar}{2 \pi} \sum_{\alpha \beta} W_{\alpha \beta} \cdot\left[\frac{\partial}{\partial \mathbf{r}_{i}} M_{\alpha \beta}\right] \\
& =\frac{\hbar}{2 \pi} \operatorname{Tr}\left[\mathbf{M}^{-1} \cdot \frac{\partial}{\partial \mathbf{r}_{i}} \mathbf{M}\right]
\end{aligned}
$$

(where we have recalled the definition $\mathbf{W}=\mathbf{M}^{-1}$ ), and inserting this into (2) we obtain the FSC formula for the Casimir force, Eq. (1b). To obtain the FSC formula for the Casimir energy, we note that the Casimir force on an object is minus the derivative of the energy with respect to a rigid displacement of that object; using the standard identity

$$
\frac{\partial}{\partial \mathbf{r}_{i}} \ln \operatorname{det} \mathbf{M}=\operatorname{Tr}\left[\mathbf{M}^{-1} \cdot \frac{\partial}{\partial \mathbf{r}_{i}} \mathbf{M}\right],
$$

and choosing the zero of energy to correspond to the energy of the configuration in which all objects are removed to infinite separations (for which configuration we denote the SIE matrix by $\mathbf{M}_{\infty}$ ), we recover Eq. (1a). Finally, Eq. (1c) follows from taking derivatives with respect to a rigid rotation instead of a rigid displacement.

This completes the stress-tensor derivation of the FSC formulas for the case of PEC objects.

\section{Stress-tensor derivation of FSC formulas for general objects}

The derivation of the FSC formulas for general objects is now a straightforward generalization of the procedure for PEC objects. Again we start with Eq. (6), and again we insert in this equation the factorized expressions for scattering DGFs that we derived in Sec. B 3; the difference is that for non-PEC objects we must now use the more complicated expressions (B22). Mimicking the discussion following Eqs. (7) above now leads to a modified version of Eq. (9) in which the $\mathcal{I}$ kernel is promoted to a $2 \times 2$ matrix:

$$
F_{i}(\xi)=+\frac{\hbar}{2 \pi} \operatorname{Tr} \sum_{\alpha \beta}\left(\begin{array}{cc}
W_{\alpha \beta}^{\mathrm{EE}} & W_{\alpha \beta}^{\mathrm{EM}} \\
W_{\alpha \beta}^{\mathrm{ME}} & W_{\alpha \beta}^{\mathrm{MM}}
\end{array}\right) \int_{\sup \mathbf{f}_{\alpha}} d \mathbf{r}_{\alpha} \int_{\sup _{\beta} \mathbf{f}_{\beta}} d \mathbf{r}_{\beta}\left\{f_{\alpha k}\left(\mathbf{r}_{\alpha}\right)\left(\begin{array}{cc}
Z_{0} Z^{e} \kappa^{e} \overline{\mathcal{I}}_{i k \ell}\left(\mathbf{r}_{\alpha}, \mathbf{r}_{\beta}\right) & \kappa^{e} \overline{\mathcal{J}}_{i p \ell}\left(\mathbf{r}_{\alpha}, \mathbf{r}_{\beta}\right) \\
-\kappa^{e} \overline{\mathcal{J}}_{i p \ell}\left(\mathbf{r}_{\alpha}, \mathbf{r}_{\beta}\right) & \frac{\kappa^{e}}{Z_{0} Z^{e}} \overline{\mathcal{I}}_{i p \ell}\left(\mathbf{r}_{\alpha}, \mathbf{r}_{\beta}\right)
\end{array}\right) f_{\beta \ell}\left(\mathbf{r}_{\beta}\right)\right\},
$$

with Tr denoting a $2 \times 2$ matrix trace and the $\overline{\mathcal{J}}$ kernel defined in analogy to $\overline{\mathcal{I}}$ :

$$
\begin{aligned}
\overline{\mathcal{J}}_{i k \ell}\left(\mathbf{r}, \mathbf{r}^{\prime}\right) \equiv & \mathcal{J}_{i k \ell}\left(\mathbf{r}, \mathbf{r}^{\prime}\right)+\mathcal{J}_{i \ell k}\left(\mathbf{r}^{\prime}, \mathbf{r}\right), \\
\mathcal{J}_{i k \ell}\left(\mathbf{r}_{\alpha}, \mathbf{r}_{\beta}\right) \equiv & \left(\kappa^{e}\right)^{2} \oint_{\mathcal{C}}\left\{G_{i k}\left(\mathbf{r}_{\alpha}, \mathbf{x}\right) C_{\ell j}\left(\mathbf{x}, \mathbf{r}_{\beta}\right)+C_{i k}\left(\mathbf{r}_{\alpha}, \mathbf{x}\right) G_{\ell j}\left(\mathbf{x}, \mathbf{r}_{\beta}\right)\right. \\
& \left.-\frac{\delta_{i j}}{2}\left[G_{m k}\left(\mathbf{r}_{\alpha}, \mathbf{x}\right) C_{\ell m}\left(\mathbf{x}, \mathbf{r}_{\beta}\right)+C_{m k}\left(\mathbf{r}_{\alpha}, \mathbf{x}\right) G_{\ell m}\left(\mathbf{x}, \mathbf{r}_{\beta}\right)\right]\right\} \hat{\mathbf{n}}_{j} d \mathbf{x} .
\end{aligned}
$$

Again in analogy to $\overline{\mathcal{I}}$, the surface integrals in the definition of $\overline{\mathcal{J}}$ may be evaluated in closed form to yield

$$
\overline{\mathcal{J}}_{i k \ell}\left(\mathbf{r}, \mathbf{r}^{\prime}\right)= \begin{cases}0, & \text { if } \quad \mathbf{r}, \mathbf{r}^{\prime} \text { lie both inside or both outside } \mathcal{C}, \\ \frac{\partial}{\partial \mathbf{r}_{i}^{i}} C_{k \ell}\left(\mathbf{r}^{\mathrm{I}}, \mathbf{r}^{\mathrm{E}}\right), & \text { if } \quad \mathbf{r}, \mathbf{r}^{\prime} \text { lie on opposite sides of } \mathcal{C},\end{cases}
$$

and, armed with (10) and (13), it is now easy to identify the integral over basis functions in (12) as nothing but the derivative of the SIE matrix:

$$
\int_{\sup \mathbf{f}_{\alpha}} d \mathbf{r}_{\alpha} \int_{\sup _{\beta} \mathbf{f}_{\beta}} d \mathbf{r}_{\beta}\left\{f_{\alpha k}\left(\mathbf{r}_{\alpha}\right)\left(\begin{array}{cc}
Z_{0} Z^{e} \kappa^{e} \overline{\mathcal{I}}_{i k \ell}\left(\mathbf{r}_{\alpha}, \mathbf{r}_{\beta}\right) & \kappa^{e} \overline{\mathcal{J}}_{i p \ell}\left(\mathbf{r}_{\alpha}, \mathbf{r}_{\beta}\right) \\
-\kappa^{e} \overline{\mathcal{J}}_{i p \ell}\left(\mathbf{r}_{\alpha}, \mathbf{r}_{\beta}\right) & \frac{\kappa^{e}}{Z_{0} Z^{e}} \overline{\mathcal{I}}_{i p \ell}\left(\mathbf{r}_{\alpha}, \mathbf{r}_{\beta}\right)
\end{array}\right) f_{\beta \ell}\left(\mathbf{r}_{\beta}\right)\right\}=\frac{\partial}{\partial \mathbf{r}_{i}}\left(\begin{array}{cc}
M_{\alpha \beta}^{\mathrm{EE}} & M_{\alpha \beta}^{\mathrm{EM}} \\
M_{\alpha \beta}^{\mathrm{ME}} & M_{\alpha \beta}^{\mathrm{MM}}
\end{array}\right) .
$$

Inserting (14) into (12) now simply reproduces Eq. (11) with the $\mathbf{M}$ matrix understood to refer to the general-material SIE matrix in Eq. (B13). 


\section{PATH-INTEGRAL DERIVATION OF THE FSC CASIMIR FORMULAS}

It is remarkable that the path-integral approach to Casimir physics, which bears little superficial resemblance to the stresstensor formalism of the previous section, may nonetheless be used to furnish a separate and entirely independent derivation of the same FSC formulas that we derived above using stress-tensor ideas. In this section we present this alternate derivation. Our method builds on existing techniques for extracting Casimir energies from constrained path integrals; Appendix D reviews this previous work to provide background and context for the discussion of this section.

The path-integral procedure presented here differs from typical path-integral treatments of Casimir phenomena in at least two ways. First, whereas many authors write the action for the electromagnetic field in terms of the gauge-independent $\mathbf{E}$ and $\mathbf{B}$ fields [2], or in terms of the four-vector potential $A^{\mu}$ in a way that depends on a specific choice of gauge (often the "temporal" or "Weyl" gauge $A^{0} \equiv 0$ [38]), here we write the action in terms of $A^{\mu}$ with a Fadeev-Popov parameter that allows arbitrary gauge choices (Sec. III A); we verify explicitly that the Fadeev-Popov parameter is absent from all final physical predictions. (This portion of our treatment is similar to that of Ref. [39].)

Second (Sec. III B), we introduce a new implementation of the constraint that the path integral extend only over field configurations satisfying the boundary conditions. Our representation emphasizes the continuity of the tangential $\mathbf{E}$ and $\mathbf{H}$ fields across the surfaces of the objects in a Casimir geometry, and the Lagrange multipliers that we introduce to enforce the constraints have an attractive physical interpretation as surface currents, thus establishing a connection to the SIE ideas reviewed above.

Combining the techniques of Secs. III A and III B yields an expression for the Casimir energy involving functional integrals over both the electromagnetic field and the surfacecurrent distributions (Sec. IIIC). After integrating out the photons, we are left with functional integrals over surface currents, with an effective action describing the interactions of these currents through the electrical media interior and exterior to the objects. Upon discretization, this action turns out to involve precisely the same surface-current-interaction matrix that appears in the SIE formulation of scattering reviewed in Appendix B, and the Casimir energy may be evaluated to yield precisely the same results obtained via the stress-tensor procedure of Sec. II.

\section{A. Euclidean Lagrangian for the electromagnetic field}

The usual (Minkowski-space) Lagrangian for the electromagnetic field is

$$
\begin{aligned}
S & =\int \frac{d \omega}{2 \pi} \int d \mathbf{x} \mathcal{L}(\omega, \mathbf{x}), \\
\mathcal{L}(\omega, \mathbf{x}) & =\frac{1}{2}\left[\epsilon(\omega, \mathbf{x})|\mathbf{E}(\omega, \mathbf{x})|^{2}-\mu(\omega, \mathbf{x})|\mathbf{H}(\omega, \mathbf{x})|^{2}\right] .
\end{aligned}
$$

Rewriting $\mathbf{E}$ and $\mathbf{H}$ in terms of the four-vector potential $A^{\mu}$, integrating by parts, and rotating to Euclidean space via the prescription $\left\{\omega, A^{0}, A^{0 *}\right\} \rightarrow\left\{i \xi, i A^{0}, i A^{0 *}\right\}$ yields a Euclidean action density of the form

$$
\begin{aligned}
\mathcal{L}_{\mathrm{E}}(\xi, \mathbf{x})= & \frac{\epsilon(i \xi, \mathbf{x})}{2}\left(-\xi^{2} A^{i *} A^{i}-i \xi A^{0 *} \partial_{i} A^{i}\right. \\
& \left.-i \xi A^{i *} \partial_{i} A^{0}+A^{0 *} \partial_{i} \partial_{i} A^{0}\right) \\
& +\frac{1}{2 \mu(i \xi, \mathbf{x})}\left(A^{i *} \partial_{j} \partial_{j} A^{i}-A^{i *} \partial_{i} \partial_{j} A^{j}\right),
\end{aligned}
$$

or, introducing a convenient matrix-vector notation,

$$
\mathcal{L}_{\mathrm{E}}^{\prime}(\xi, \mathbf{x})=\frac{1}{2}\left(\begin{array}{c}
\mathcal{A}^{0} \\
\mathcal{A}^{1} \\
\mathcal{A}^{2} \\
\mathcal{A}^{3}
\end{array}\right)^{\dagger}\left[\mathfrak{D}_{1}(\xi)-\mathfrak{D}_{2}(\xi)\right]\left(\begin{array}{c}
\mathcal{A}^{0} \\
\mathcal{A}^{1} \\
\mathcal{A}^{2} \\
\mathcal{A}^{3}
\end{array}\right)
$$

where we have defined

$$
\left(\begin{array}{c}
\mathcal{A}^{0} \\
\mathcal{A}^{1} \\
\mathcal{A}^{2} \\
\mathcal{A}^{3}
\end{array}\right)=\left(\begin{array}{c}
\sqrt{\epsilon \mu} A^{0} \\
A^{1} \\
A^{2} \\
A^{3}
\end{array}\right)
$$

and

$$
\mathfrak{D}_{1}=\left(\begin{array}{cccc}
-\epsilon \xi^{2}+\frac{1}{\mu} \nabla^{2} & 0 & 0 & 0 \\
0 & -\epsilon \xi^{2}+\frac{1}{\mu} \nabla^{2} & 0 & 0 \\
0 & 0 & -\epsilon \xi^{2}+\frac{1}{\mu} \nabla^{2} & 0 \\
0 & 0 & 0 & -\epsilon \xi^{2}+\frac{1}{\mu} \nabla^{2}
\end{array}\right), \quad \mathfrak{D}_{2}=\left(\begin{array}{cccc}
-\epsilon \xi^{2} & i \sqrt{\frac{\epsilon}{\mu} \xi \partial_{x}} & i \sqrt{\frac{\epsilon}{\mu} \xi \partial_{y}} & i \sqrt{\frac{\epsilon}{\mu}} \xi \partial_{z} \\
i \sqrt{\frac{\epsilon}{\mu}} \xi \partial_{x} & \frac{1}{\mu} \partial_{x}^{2} & \frac{1}{\mu} \partial_{x} \partial_{y} & \frac{1}{\mu} \partial_{x} \partial_{z} \\
i \sqrt{\frac{\epsilon}{\mu}} \xi \partial_{y} & \frac{1}{\mu} \partial_{y} \partial_{x} & \frac{1}{\mu} \partial_{y}^{2} & \frac{1}{\mu} \partial_{y} \partial_{z} \\
i \sqrt{\frac{\epsilon}{\mu}} \xi \partial_{z} & \frac{1}{\mu} \partial_{z} \partial_{x} & \frac{1}{\mu} \partial_{z} \partial_{y} & \frac{1}{\mu} \partial_{z}^{2}
\end{array}\right) .
$$

The four-vector field $\mathcal{A}^{\mu}$ defined by (16) will be the field over which we path integrate, and Eq. (15) is almost, but not quite, the quantity that enters into the exponent of the constrained path-integral expression for the Casimir energy, Eq. (D3). To complete the story, we must add a Fadeev-Popov gauge fixing term, which we do in analogy to the usual QED procedure [40] by simply displacing the coefficient of $\mathfrak{D}_{2}$ term in (15) away from unity to ensure that the matrix in square brackets has no zero eigenvalues. Our final Euclidean action is

$$
\mathcal{L}_{\mathrm{E}}(\xi, \mathbf{x})=\mathcal{A}^{\mu}\left[\mathfrak{D}_{1}(\xi)-\left(1-\frac{1}{\alpha_{\mathrm{FP}}}\right) \mathfrak{D}_{2}(\xi)\right]_{\mu \nu} \mathcal{A}^{v} \equiv \mathcal{A} \cdot \mathfrak{D}(\xi) \cdot \mathcal{A}
$$


where the Faddeev-Popov gauge-choice parameter $\alpha_{\mathrm{FP}}$ may be chosen to have any finite value and is absent from all final physical predictions, as explicitly verified below [see Eqs. (34) and (35)]. Following the general procedure reviewed in Appendix D, we can now write the Casimir energy at inverse temperature $\beta$ in the form

$$
\begin{gathered}
\mathcal{E}=-\frac{1}{\beta} \ln \frac{Z(\beta)}{Z_{\infty}(\beta)}=-\frac{1}{\beta} \sum_{n=0}^{\infty} \ln \frac{\mathcal{Z}\left(\beta, \xi_{n}\right)}{\mathcal{Z}_{\infty}\left(\beta, \xi_{n}\right)}, \\
\mathcal{Z}(\beta, \xi)=\int\left[\mathcal{D} \mathcal{A}^{\mu}\right]_{\mathrm{C}} e^{-\frac{\beta}{2} \int \mathcal{A} \cdot \mathcal{D}(\xi) \cdot \mathcal{A} d \mathbf{x}},
\end{gathered}
$$

with the notation $[\cdots]_{C}$ indicating that the functional integration ranges only over field configurations that satisfy the boundary conditions in the presence of our interacting material objects.

\section{B. Boundary conditions enforced by surface-current Lagrange multipliers}

In a scattering geometry consisting of one or more homogeneous bodies bodies embedded in a homogeneous medium, the boundary conditions on the electromagnetic field are simply that the tangential $\mathbf{E}$ and $\mathbf{H}$ fields be continuous across all material boundaries: If $\mathbf{x}$ is a point on the surface of an object, then we require

$$
\begin{aligned}
\mathbf{t}^{a} \cdot\left[\mathbf{E}^{\text {in }}(\mathbf{x})-\mathbf{E}^{\text {out }}(\mathbf{x})\right] & =0, \\
\mathbf{t}^{a} \cdot\left[\mathbf{H}^{\text {in }}(\mathbf{x})-\mathbf{H}^{\text {out }}(\mathbf{x})\right] & =0,
\end{aligned}
$$

where $\{\mathbf{E}, \mathbf{H}\}^{\text {in,out }}$ are the fields evaluated just inside and just outside the object surface at $\mathbf{x}$, and where $\mathbf{t}^{a}(a \in\{1,2\})$ are vectors tangent to the surface at $\mathbf{x}$ [Fig. 1(a)]. In terms of the modified four-vector potential $\mathcal{A}^{\mu}$, these conditions may be written in the form

$$
\begin{aligned}
t_{i}^{a}\left\{L_{i \mu}^{\mathrm{E}, \text { in }}(\mathbf{x})-L_{i \mu}^{\mathrm{E}, \text { out }}(\mathbf{x})\right\} \mathcal{A}^{\mu}(\mathbf{x}) & =0, \\
t_{i}^{a}\left\{L_{i \mu}^{\mathrm{M}, \text { in }}(\mathbf{x})-L_{i \mu}^{\mathrm{M}, \mathrm{out}}(\mathbf{x})\right\} \mathcal{A}^{\mu}(\mathbf{x}) & =0,
\end{aligned}
$$

where $\mathbf{L}^{\mathrm{E}, r}$ and $\mathbf{L}^{\mathrm{M}, r}$ are differential operators that operate on $\mathcal{A}^{\mu}$ to yield the components of the $\mathbf{E}$ and $\mathbf{H}$ fields in region $r$. (We are here using a shorthand in which the $A^{\mu}$ fields in the different regions, $\mathcal{A}^{\mu, \text { in }}$ and $\mathcal{A}^{\mu, \text { out }}$, are abbreviated simply as $\mathcal{A}^{\mu}$ and pulled outside the braces.) In a homogeneous region with spatially constant relative permittivity and permeability $\epsilon(\xi, \mathbf{x})=\epsilon^{r}(\xi), \mu(\xi, \mathbf{x})=\mu^{r}(\xi)$, the $\mathbf{L}$ operators take the form

$$
\begin{aligned}
\mathbf{L}^{\mathrm{E}, r} & =\left(\begin{array}{cccc}
-\frac{1}{\sqrt{\epsilon^{r} \mu^{r}}} \partial_{x} & i \xi & 0 & 0 \\
-\frac{1}{\sqrt{\epsilon^{r} \mu^{r}}} \partial_{y} & 0 & i \xi & 0 \\
-\frac{1}{\sqrt{\epsilon^{r} \mu^{r}}} \partial_{z} & 0 & 0 & i \xi
\end{array}\right), \\
\mathbf{L}^{\mathrm{M}, r} & =\frac{1}{\mu^{r}}\left(\begin{array}{cccc}
0 & 0 & -\partial_{z} & \partial_{y} \\
0 & \partial_{z} & 0 & -\partial_{x} \\
0 & -\partial_{y} & \partial_{x} & 0
\end{array}\right) .
\end{aligned}
$$

Equations (21) are a set of four boundary conditions for each point $\mathbf{x}$ on the surfaces of the material bodies in our geometry; in the language of Appendix D, these are our constraints $L_{\alpha} \phi$, and to each constraint we now associate a Lagrange multiplier. We use the symbols $K^{a}(\mathbf{x})$ and $N^{a}(\mathbf{x})(a=1,2)$, respectively, to denote the Lagrange multipliers associated with constraints (21a) and (21b) at the single point $\mathbf{x}$ [Fig. 1(b)]. Then the $\delta$ functions that enforce the boundary conditions (21) at $\mathbf{x}$ are

$$
\begin{gathered}
\delta\left(\mathbf{E}_{\|}^{\text {in }}(\mathbf{x})-\mathbf{E}_{\|}^{\text {out }}(\mathbf{x})\right)=\int \frac{d \mathbf{K}_{\mathbf{x}}}{(2 \pi)^{2}} e^{i \mathbf{K}_{\mathbf{x}} \cdot\left[\mathbf{L}_{\mu}^{\mathrm{E} \text {,in }}-\mathbf{L}_{\mu}^{\text {E.out }}\right] \mathcal{A}^{\mu}(\mathbf{x})}, \\
\delta\left(\mathbf{H}_{\|}^{\text {in }}(\mathbf{x})-\mathbf{H}_{\|}^{\text {out }}(\mathbf{x})\right)=\int \frac{d \mathbf{N}_{\mathbf{x}}}{(2 \pi)^{2}} e^{i \mathbf{N}_{\mathbf{x}} \cdot\left[\mathbf{L}_{\mu}^{\text {,in }}-\mathbf{L}_{\mu}^{\mathrm{M}, \text { out }}\right] \mathcal{A}^{\mu}(\mathbf{x})},
\end{gathered}
$$

where we may think of $\left\{\mathbf{K}_{\mathbf{x}}, \mathbf{N}_{\mathbf{x}}\right\}=\sum_{a=1}^{2}\left\{K^{a}, N^{a}\right\} \mathbf{t}^{a}$ as vectors in the tangent space to the boundary surface at $\mathbf{x}$. Aggregating the corresponding $\delta$ functions for all points on the surface of a single object, we obtain functional $\delta$ functions,

$$
\begin{aligned}
& \int \mathcal{D} \mathbf{K}(\mathbf{x}) e^{i \int_{\partial \mathcal{O}} \mathbf{K}(\mathbf{x}) \cdot\left[\mathbf{L}_{\mu}^{\mathrm{E}, \mathrm{in}}-\mathbf{L}_{\mu}^{\mathrm{E}, \text { out }}\right] \mathcal{A}^{\mu}(\mathbf{x}) d \mathbf{x},} \\
& \int \mathcal{D} \mathbf{N}(\mathbf{x}) e^{i \int_{\partial \mathcal{O}} \mathbf{N}(\mathbf{x}) \cdot\left[\mathbf{L}_{\mu}^{\mathrm{M}, \mathrm{in}}-\mathbf{L}_{\mu}^{\mathrm{M}, \text { out }}\right] \mathcal{A}^{\mu}(\mathbf{x}) d \mathbf{x}},
\end{aligned}
$$

where the integral in the exponent is over the surface $\partial \mathcal{O}$ of an object in our geometry, and where the functional integrations $\int \mathcal{D} \mathbf{K}, \int \mathcal{D} \mathbf{N}$ extend over all possible tangential vector fields on $\partial \mathcal{O}$.

Since $\mathbf{K}$ and $\mathbf{N}$ are tangential vector fields on $\partial \mathcal{O}$ that enforce the continuity of the tangential electric and magnetic fields, respectively, it is tempting to interpret these quantities as electric and magnetic surface current densities, and with their introduction our path-integral formalism begins to exhibit the first glimmers of resemblance to the SIE picture reviewed in Appendix B.

\section{Evaluation of the constrained path integral}

In general we have one copy of the functional $\delta$ functions (24) for the surface of each object in our geometry. Let $\left\{\mathbf{K}_{r}, \mathbf{N}_{r}\right\}$ denote the Lagrange-multiplier distributions on the surface of the $r$ th object; the constrained path integral then reads

$$
\begin{aligned}
\mathcal{Z}(\beta, \xi)= & \int\left[\mathcal{D} \mathcal{A}^{\mu}\right]_{C} e^{-\frac{\beta}{2} \int \mathcal{A} \cdot \mathfrak{D} \cdot \mathcal{A} d \mathbf{x}} \\
= & \int \prod_{r} \mathcal{D} \mathbf{K}_{r} \mathcal{D} \mathbf{N}_{r} \int \mathcal{D} \mathcal{A}^{\mu}\left\{e^{-\frac{\beta}{2} \int \mathcal{A} \cdot \mathfrak{D} \cdot \mathcal{A} d \mathbf{x}}\right. \\
& \left.\times e^{+i \sum_{r} \int_{\partial \mathcal{O}_{r}}\left\{\mathbf{K}_{r} \cdot\left(\mathbf{L}^{\mathrm{E}, r}-\mathbf{L}^{\mathrm{E}, e}\right)+\mathbf{N}_{r} \cdot\left(\mathbf{L}^{\mathrm{M}, r}-\mathbf{L}^{\mathrm{M}, e}\right)\right\} \cdot \mathcal{A} d \mathbf{x}}\right\},
\end{aligned}
$$




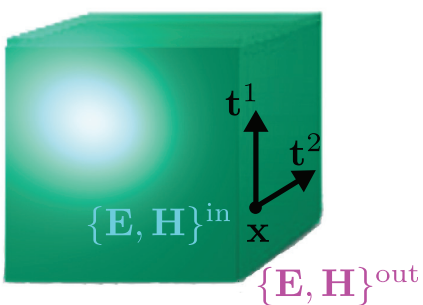

(a)

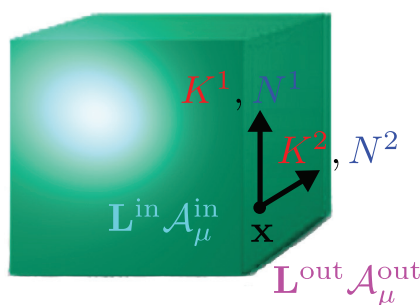

(b)

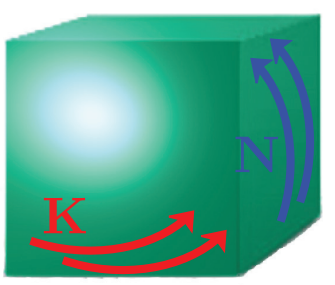

(c)

FIG. 1. (Color online) Enforcing boundary conditions via surface-current Lagrange multipliers. (a) Consider a single point $\mathbf{x}$ on the surface of an object in a Casimir geometry. The boundary conditions at $\mathbf{x}$, which must be satisfied in the constrained path integral (19), are that the tangential components of the $\mathbf{E}$ and $\mathbf{H}$ fields be continuous as we pass from inside to outside the object [Eq. (20)]; here $\left\{\mathbf{t}^{1}, \mathbf{t}^{2}\right\}$ are vectors tangent to the surface at $\mathbf{x}$. (b) We rewrite the boundary conditions in terms of differential operators $\mathbf{L}^{\mathrm{E}, \mathrm{M}}$ operating on the $\mathcal{A}$ field, and we introduce Lagrange multipliers $\left\{K^{1}, K^{2}, N^{1}, N^{2}\right\}$ to enforce the boundary conditions at $\mathbf{x}$; specifically, $K^{1}, K^{2}$ enforce the tangential E-field continuity at $\mathbf{x}$, while $N^{1}, N^{2}$ enforce the tangential $\mathbf{H}$-field continuity [Eq. (23)]. (c) Repeating this procedure for all points on the object surface, we obtain Lagrange multiplier fields $\mathbf{K}(\mathbf{x}), \mathbf{N}(\mathbf{x})$, which have an obvious interpretation as the electric and magnetic surface currents of Fig. 4. Integrating the photon field out of the path integral then yields an effective action describing the interactions of these surface currents [Eqs. (28), (41), and (42)], leading ultimately to our FSC formulas for the Casimir energy.

with $\int_{\partial \mathcal{O}_{r}}$ denoting integration over the surface of object $r$ and with the path integration over $\mathcal{A}$ in the second line now unconstrained [compare Eq. (D6)]. This is just a standard Gaussian functional integral, which we proceed to evaluate using standard techniques [40,41].

To this end, it is convenient to think of breaking up the functional integration over $\mathcal{A}^{\mu}$ into separate integrations over the fields in each object and in the exterior region,

$$
\int \mathcal{D} \mathcal{A}^{\mu}=\int \mathcal{D} \mathcal{A}_{e}^{\mu} \prod_{r} \mathcal{D} \mathcal{A}_{r}^{\mu}
$$

where $\mathcal{A}_{r}^{\mu}$ is the field in the interior of region $r$ and $\mathcal{A}_{e}^{\mu}$ is the field in the exterior region. The matrix $\mathfrak{D}(\mathbf{x})$, which depends on $\mathbf{x}$ through $\epsilon$ and $\mu$, is constant in each region due to the piecewise homogeneity of the geometry, while the operators $L^{\mathrm{EM}, r}$ only operate on the fields in region $r$. The functional integral becomes

$$
\begin{aligned}
& \mathcal{Z}(\beta, \xi) \\
& =\int \prod_{r} \mathcal{D} \mathbf{K}_{r} \mathcal{D} \mathbf{N}_{r} \int \mathcal{D} \mathcal{A}_{e}^{\mu} \prod_{r} \mathcal{D} \mathcal{A}_{r}^{\mu} \\
& \quad \times\left\{e^{-\frac{\beta}{2} \int_{V_{e}} \mathcal{A}_{e} \cdot \mathcal{D}_{e} \cdot \mathcal{A}_{e}-\frac{\beta}{2} \sum_{r} \int_{V_{r}} \mathcal{A}_{r} \cdot \mathcal{D}_{r} \cdot \mathcal{A}_{r}}\right. \\
& \left.\quad \times e^{\left.+i \sum_{r} \int_{\partial_{\mho_{r}}}\left\{\mathbf{K}_{r} \cdot \mathbf{L}^{\mathrm{E}, r}+\mathbf{N}_{r} \cdot \mathbf{L}^{\mathrm{M}, r}\right] \cdot \mathcal{A}_{r}-\left[\mathbf{K}_{r} \cdot \mathbf{L}^{\mathrm{E}, e}+\mathbf{N}_{r} \cdot \mathbf{L}^{\mathrm{M}, e}\right] \cdot \mathcal{A}_{e}\right\} d \mathbf{x}}\right\},
\end{aligned}
$$

with $\int_{V_{r}}$ denoting volume integration over the interior of region $r$. Now performing the Gaussian functional integrations over the fields $\mathcal{A}_{r}^{\mu}$ immediately yields an expression of the form (D8),

$$
\mathcal{Z}(\beta, \xi)=\{\#\} \int \prod_{r} \mathcal{D} \mathbf{K}_{r} \mathcal{D} \mathbf{N}_{r} e^{-\frac{1}{\beta} S^{\text {eff }}},
$$

where $\{\#\}$ is an unimportant constant that cancels upon taking the ratio in (18) (and which is omitted from the equations below) and where the effective action for the surface currents,

$$
S^{\mathrm{eff}}=\sum_{r=1}^{R} S_{r}\left[\mathbf{K}_{r}, \mathbf{N}_{r}\right]+S_{e}\left[\left\{\mathbf{K}_{r}, \mathbf{N}_{r}\right\}_{r=1}^{R}\right],
$$

contains terms describing both the self-interactions and the mutual interactions of currents on the object surfaces, as we now discuss.

Consider first what happens when we integrate $\mathcal{A}_{r}^{\mu}$ out of (26). Because the exponent of (26) couples $\mathcal{A}_{r}^{\mu}$ only to $\mathbf{K}_{r}, \mathbf{N}_{r}$ (and not to currents on other objects $r^{\prime} \neq r$ ), integrating out $\mathcal{A}_{r}^{\mu}$ yields an effective action involving only $\mathbf{K}_{r}, \mathbf{N}_{r}$ :

$$
\begin{aligned}
\int & \mathcal{D} \mathcal{A}_{r}^{\mu} e^{-\frac{\beta}{2} \int \mathcal{A}_{r} \cdot \mathcal{D}_{r} \cdot \mathcal{A}_{r}+i \int_{\partial \partial_{r}}\left[\mathbf{K}_{r} \cdot \mathbf{L}^{\mathrm{E}, r}+\mathbf{N}_{r} \cdot \mathbf{L}^{\mathrm{M}, r}\right] \cdot \mathcal{A}_{r}} \\
= & e^{-\frac{1}{2 \beta} \iint_{\partial O_{r}}\left[\mathbf{K}_{r} \cdot \mathbf{L}^{\mathrm{E}, r}+\mathbf{N}_{r} \cdot \mathbf{L}^{\mathrm{M}, r}\right] \cdot \mathcal{D}_{1}^{-1} \cdot\left[\mathbf{K}_{r} \cdot \mathbf{L}^{\mathrm{E}, r}+\mathbf{N}_{r} \cdot \mathbf{L}^{\mathrm{M}, r}\right]} \\
& \equiv e^{-\frac{1}{\beta} S_{r}\left[\mathbf{K}_{r}, \mathbf{N}_{r}\right]}
\end{aligned}
$$

The effective action $S_{r}$ describes the self-interactions of electric and magnetic currents on $\partial \mathcal{O}_{r}$ mediated by virtual photons propagating through the interior of object $r$. More precisely, we have

$$
\begin{aligned}
S_{r}\left[\mathbf{K}_{r}, \mathbf{N}_{r}\right]= & \frac{1}{2} \int_{\partial \mathcal{O}_{r}} d \mathbf{x} \int_{\partial \mathcal{O}_{r}} d \mathbf{x}^{\prime}\left\{\mathbf{K}_{r}(\mathbf{x}) \cdot \boldsymbol{\gamma}^{\mathrm{EE}, \mathrm{r}}\left(\mathbf{x}, \mathbf{x}^{\prime}\right) \cdot \mathbf{K}_{r}\left(\mathbf{x}^{\prime}\right)\right. \\
& +\mathbf{K}_{r}(\mathbf{x}) \cdot \boldsymbol{\gamma}^{\mathrm{EM}, \mathrm{r}}\left(\mathbf{x}, \mathbf{x}^{\prime}\right) \cdot \mathbf{N}_{r}\left(\mathbf{x}^{\prime}\right) \\
& +\mathbf{N}_{r}(\mathbf{x}) \cdot \boldsymbol{\gamma}^{\mathrm{ME}, \mathrm{r}}\left(\mathbf{x}, \mathbf{x}^{\prime}\right) \cdot \mathbf{K}_{r}\left(\mathbf{x}^{\prime}\right) \\
& \left.+\mathbf{N}_{r}(\mathbf{x}) \cdot \boldsymbol{\gamma}^{\mathrm{MM}, \mathrm{r}}\left(\mathbf{x}, \mathbf{x}^{\prime}\right) \cdot \mathbf{N}_{r}\left(\mathbf{x}^{\prime}\right)\right\}
\end{aligned}
$$

with the components of the tensor kernels given by (P,Q $\in$ $\{\mathrm{E}, \mathrm{H}\})$

$$
\boldsymbol{\gamma}_{i j}^{\mathrm{PQ}, \mathrm{r}}=L_{i \mu}^{\mathrm{P}, r} \mathfrak{D}_{r \mu \nu}^{-1} L_{j \nu}^{\mathrm{Q}, r} .
$$

We will see presently that the $\boldsymbol{\gamma}^{\mathrm{PQ}, \mathrm{r}}$ matrices here turn out to be nothing but the usual dyadic Green's tensors $\Gamma^{\mathrm{PQ}, \mathrm{r}}$ for the homogeneous medium inside object $\mathcal{O}_{r}$. 
To see this, it is easiest to perform the matrix multiplications of Eq. (30) in momentum space, where we have

$$
\begin{aligned}
\mathbf{L}^{\mathrm{E}, r} & =\left(\begin{array}{cccc}
-i \frac{1}{\sqrt{\epsilon^{r} \mu^{r}}} k_{x} & i \xi & 0 & 0 \\
-i \frac{1}{\sqrt{\epsilon^{r} \mu^{r}}} k_{y} & 0 & i \xi & 0 \\
-i \frac{1}{\sqrt{\epsilon^{r} \mu^{r}}} k_{z} & 0 & 0 & i \xi
\end{array}\right), \\
\mathbf{L}^{\mathrm{M}, r} & =\frac{1}{\mu^{r}}\left(\begin{array}{cccc}
0 & 0 & -i k_{z} & i k_{y} \\
0 & i k_{z} & 0 & -i k_{x} \\
0 & -i k_{y} & i k_{x} & 0
\end{array}\right),
\end{aligned}
$$

and [cf. Eq. (17)]

$$
\begin{aligned}
\mathfrak{D}_{r}^{-1} & =\left[\mathfrak{D}_{r, 1}-\left(1-\frac{1}{\alpha^{\mathrm{FP}}}\right) \mathfrak{D}_{r, 2}\right]^{-1} \\
& =-\left[\frac{1}{\mu^{r}}\left(\kappa^{2}+|\mathbf{k}|^{2}\right) \mathbf{1}-\left(1-\frac{1}{\alpha_{\mathrm{FP}}}\right) \mathfrak{D}_{r, 2}\right]^{-1} \\
& =-\frac{\mu^{r}}{\kappa^{2}+|\mathbf{k}|^{2}}\left[\mathbf{1}+\mu^{r}\left(\frac{1-\alpha_{\mathrm{FP}}}{\kappa^{2}+|\mathbf{k}|^{2}}\right) \mathfrak{D}_{r, 2}\right],
\end{aligned}
$$

where $\mathbf{1}$ is the $4 \times 4$ unit matrix, $\left\{\epsilon^{r}, \mu^{r}\right\}$ are the (spatially constant) permittivity and permeability of object $r$ at imaginary frequency $\xi, \kappa=\sqrt{\epsilon^{r} \mu^{r}} \xi$, and the momentum-space form of the $\mathfrak{D}_{r, 2}$ matrix is

$$
\mathfrak{D}_{r, 2}=-\frac{1}{\mu^{r}}\left(\begin{array}{cccc}
\kappa^{2} & \kappa k_{x} & \kappa k_{y} & \kappa k_{z} \\
\kappa k_{x} & k_{x}^{2} & k_{x} k_{y} & k_{x} k_{z} \\
\kappa k_{y} & k_{y} k_{x} & k_{y}^{2} & k_{y} k_{z} \\
\kappa k_{z} & k_{z} k_{x} & k_{z} k_{y} & k_{z}^{2}
\end{array}\right) .
$$

(The passage from the second to the third line of Eq. (32) is a standard algebraic manipulation in quantum field theory; see, e.g., Eq. (9.58) of Ref. [40]. The fact that we obtain such a concise form for the inverse of the matrix in square brackets in (31) is due to the fact that $\mathfrak{D}_{r, 2}$ is a rank 1 matrix and is known in numerical analysis as the Sherman-Morrison formula [42].)

We now note the crucial fact that the second term in (32) makes no contribution to the effective action for the surface currents. Indeed, the contribution of this term to the $\boldsymbol{\gamma}$ kernels in (30) involves triple matrix products of the form $\mathbf{L}^{\mathrm{P}, r} \cdot \mathfrak{D}_{r, 2}$. $\left(\mathbf{L}^{\mathrm{Q}, r}\right)^{\mathrm{T}}$; but an explicit calculation reveals that $\left(c=1 / \sqrt{\epsilon^{r} \mu^{r}}\right)$

$$
\begin{aligned}
\mathbf{L}^{\mathrm{E}, r} \cdot \mathfrak{D}_{r, 2} \cdot\left(\mathbf{L}^{\mathrm{E}, r}\right)^{\mathrm{T}}= & \frac{1}{\mu^{r}}\left(\begin{array}{cccc}
-i c k_{x} & i \xi & 0 & 0 \\
-i c k_{y} & 0 & i \xi & 0 \\
-i c k_{z} & 0 & 0 & i \xi
\end{array}\right) \\
& \cdot\left(\begin{array}{cccc}
\kappa^{2} & \kappa k_{x} & \kappa k_{y} & \kappa k_{z} \\
\kappa k_{x} & k_{x}^{2} & k_{x} k_{y} & k_{x} k_{z} \\
\kappa k_{y} & k_{y} k_{x} & k_{y}^{2} & k_{y} k_{z} \\
\kappa k_{z} & k_{z} k_{x} & k_{z} k_{y} & k_{z}^{2}
\end{array}\right) \\
& \cdot\left(\begin{array}{cccc}
-i c k_{x} & -i c k_{y} & -i c k_{z} \\
i \xi & 0 & 0 \\
0 & i \xi & 0 \\
0 & 0 & i \xi
\end{array}\right)
\end{aligned}
$$

$$
=\left(\begin{array}{lll}
0 & 0 & 0 \\
0 & 0 & 0 \\
0 & 0 & 0
\end{array}\right),
$$

and the other three possible $\mathbf{L} \cdot \mathfrak{D} \cdot \mathbf{L}$ products also vanish identically:

$$
\begin{aligned}
\mathbf{L}^{\mathrm{E}, r} \cdot \mathfrak{D}_{r, 2} \cdot\left(\mathbf{L}^{\mathrm{M}, r}\right)^{\mathrm{T}} & =\mathbf{L}^{\mathrm{M}, r} \cdot \mathfrak{D}_{r, 2} \cdot\left(\mathbf{L}^{\mathrm{E}, r}\right)^{\mathrm{T}} \\
& =\mathbf{L}^{\mathrm{M}, r} \cdot \mathfrak{D}_{r, 2} \cdot\left(\mathbf{L}^{\mathrm{M}, r}\right)^{\mathrm{T}}=0 .
\end{aligned}
$$

This furnishes the promised demonstration that the gaugechoice parameter $\alpha_{\mathrm{FP}}$ makes no appearance in the effective action (29), thus explicitly confirming the gauge invariance of our procedure.

Having verified that only the first term in (30) contributes to the $\boldsymbol{\gamma}$ kernels in (30), these kernels are now easy to evaluate. First,

$$
\begin{aligned}
\boldsymbol{\gamma}^{\mathrm{EE}, r}= & -\frac{\mu^{r}}{\kappa^{2}+|\mathbf{k}|^{2}}\left[\mathbf{L}^{\mathrm{E}, r} \cdot \mathbf{1} \cdot\left(\mathbf{L}^{\mathrm{E}, r}\right)^{\mathrm{T}}\right] \\
= & -\frac{\mu^{r}}{\kappa^{2}+|\mathbf{k}|^{2}}\left(\begin{array}{cccc}
-i c k_{x} & i \xi & 0 & 0 \\
-i c k_{y} & 0 & i \xi & 0 \\
-i c k_{z} & 0 & 0 & i \xi
\end{array}\right) \\
& \cdot\left(\begin{array}{ccc}
-i c k_{x} & -i c k_{y} & -i c k_{z} \\
i \xi & 0 & 0 \\
0 & i \xi & 0 \\
0 & 0 & i \xi
\end{array}\right) \\
= & \xi \frac{Z_{0} Z^{r}}{\kappa\left(\kappa^{2}+|\mathbf{k}|^{2}\right)}\left[\begin{array}{ccc}
\kappa^{2} & 0 & 0 \\
0 & \kappa^{2} & 0 \\
0 & 0 & \kappa^{2}
\end{array}\right) \\
& \left.+\left(\begin{array}{ccc}
k_{x}^{2} & k_{x} k_{y} & k_{x} k_{z} \\
k_{y} k_{x} & k_{y}^{2} & k_{y} k_{z} \\
k_{z} k_{x} & k_{z} k_{y} & k_{z}^{2}
\end{array}\right)\right] .
\end{aligned}
$$

However, a quick comparison with the momentum-space forms of the DGFs in Appendix A reveals this to be nothing but $\xi$ times the electric-electric DGF for $\mathcal{O}_{r}$, i.e.,

$$
\boldsymbol{\gamma}^{\mathrm{EE}, r}(\xi ; \mathbf{k})=\xi \cdot \boldsymbol{\Gamma}^{\mathrm{EE}, r}(\xi ; \mathbf{k}),
$$

or, transforming back to real space,

$$
\boldsymbol{\gamma}^{\mathrm{EE}, r}\left(\xi ; \mathbf{x}, \mathbf{x}^{\prime}\right)=\xi \cdot \Gamma^{\mathrm{EE}, r}\left(\xi ; \mathbf{x}, \mathbf{x}^{\prime}\right),
$$

and the connection of our formalism to SIE methodology begins to come into even sharper relief.

Next,

$$
\begin{aligned}
\boldsymbol{\gamma}^{\mathrm{EM}, r}= & -\frac{\mu^{r}}{\kappa^{2}+|\mathbf{k}|^{2}}\left[\mathbf{L}^{\mathrm{E}, r} \cdot \mathbf{1} \cdot\left(\mathbf{L}^{\mathrm{M}, r}\right)^{\mathrm{T}}\right] \\
= & -\frac{1}{\kappa^{2}+|\mathbf{k}|^{2}}\left(\begin{array}{cccc}
-i c k_{x} & i \xi & 0 & 0 \\
-i c k_{y} & 0 & i \xi & 0 \\
-i c k_{z} & 0 & 0 & i \xi
\end{array}\right) \\
& \cdot\left(\begin{array}{ccc}
0 & 0 & 0 \\
0 & i k_{z} & -i k_{y} \\
-i k_{z} & 0 & i k_{x} \\
i k_{y} & -i k_{x} & 0
\end{array}\right)
\end{aligned}
$$




$$
=-\frac{\xi}{\kappa^{2}+|\mathbf{k}|^{2}}\left(\begin{array}{ccc}
0 & -k_{z} & k_{y} \\
k_{z} & 0 & -k_{x} \\
-k_{y} & k_{x} & 0
\end{array}\right),
$$

and again comparing with Appendix A reveals that we have simply

$$
\boldsymbol{\gamma}^{\mathrm{EM}, r}=\xi \cdot \boldsymbol{\Gamma}^{\mathrm{EM}, r} .
$$

Having established the obvious pattern, it is now a short step to confirm that the remaining two cases of the $\gamma$ kernel in (30) are simply [43]

$$
\begin{aligned}
\boldsymbol{\gamma}^{\mathrm{ME}, r} & =\xi \cdot \boldsymbol{\Gamma}^{\mathrm{ME}, r}, \\
\boldsymbol{\gamma}^{\mathrm{MM}, r} & =\xi \cdot \boldsymbol{\Gamma}^{\mathrm{MM}, r},
\end{aligned}
$$

and Eq. (29), the portion of the effective action for surface currents that arises from integrating the photon field in the interior of object $\mathcal{O}_{r}$ out of (26), reads

$$
\begin{aligned}
S_{r}\left[\mathbf{K}_{r}, \mathbf{N}_{r}\right]= & \frac{\xi}{2} \int_{\partial \mathcal{O}_{r}} d \mathbf{x} \int_{\partial \mathcal{O} r} d \mathbf{x}^{\prime}\left(\begin{array}{c}
\mathbf{K}_{r}(\mathbf{x}) \\
\mathbf{N}_{r}(\mathbf{x})
\end{array}\right) \\
& \cdot\left(\begin{array}{ll}
\boldsymbol{\Gamma}^{\mathrm{EE}, r} & \boldsymbol{\Gamma}^{\mathrm{EM}, r} \\
\boldsymbol{\Gamma}^{\mathrm{ME}, r} & \boldsymbol{\Gamma}^{\mathrm{MM}, r}
\end{array}\right) \cdot\left(\begin{array}{l}
\mathbf{K}_{r}\left(\mathbf{x}^{\prime}\right) \\
\mathbf{N}_{r}\left(\mathbf{x}^{\prime}\right)
\end{array}\right) .
\end{aligned}
$$

Next, we consider integrating the photon field in the exterior region $\left(\mathcal{A}_{e}^{\mu}\right)$ out of Eq. (26). Although the computations proceed exactly as before, the the resulting contribution to the effective action is slightly more complicated. Because the exponent of (26) contains terms that couple $\mathcal{A}_{e}^{\mu}$ to the currents on all object surfaces (unlike $\mathcal{A}_{r}^{\mu}$, which couples only to surface currents on the single object $\mathcal{O}_{r}$ ), the result of integrating out $\mathcal{A}_{e}^{\mu}$ will be an effective action describing the interactions of surface currents on all object surfaces mediated by exchange of virtual photons propagating through the external medium:

$$
S_{e}\left[\left\{\mathbf{K}_{r}, \mathbf{N}_{r}\right\}\right]=\frac{\xi}{2} \sum_{r, r^{\prime}} \int_{\partial \mathcal{O}_{r}} d \mathbf{x} \int_{\partial \mathcal{O}_{r^{\prime}}} d \mathbf{x}^{\prime}\left(\begin{array}{c}
\mathbf{K}_{r}(\mathbf{x}) \\
\mathbf{N}_{r}(\mathbf{x})
\end{array}\right) \cdot\left(\begin{array}{cc}
\Gamma^{\mathrm{EE}, e} & \boldsymbol{\Gamma}^{\mathrm{EM}, e} \\
\Gamma^{\mathrm{ME}, e} & \boldsymbol{\Gamma}^{\mathrm{MM}, e}
\end{array}\right) \cdot\left(\begin{array}{c}
\mathbf{K}_{r^{\prime}}\left(\mathbf{x}^{\prime}\right) \\
\mathbf{N}_{r^{\prime}}\left(\mathbf{x}^{\prime}\right)
\end{array}\right) .
$$

Combining (41) and (42), the full path integral (27) now reads, for the particular case of two objects,

$$
\mathcal{Z}(\beta, \xi)=\int \mathcal{D} \mathbf{K}_{i} \mathcal{D} \mathbf{N}_{i} \exp \left\{-\frac{\xi}{2 \beta} \int\left(\begin{array}{l}
\mathbf{K}_{1} \\
\mathbf{N}_{1} \\
\mathbf{K}_{2} \\
\mathbf{N}_{2}
\end{array}\right)^{\mathrm{T}} \cdot(\Gamma) \cdot\left(\begin{array}{l}
\mathbf{K}_{1} \\
\mathbf{N}_{1} \\
\mathbf{K}_{2} \\
\mathbf{N}_{2}
\end{array}\right)\right\},
$$

with

$$
(\Gamma)=\left(\begin{array}{cccc}
\boldsymbol{\Gamma}^{\mathrm{EE}, 1}+\Gamma^{\mathrm{EE}, e} & \boldsymbol{\Gamma}^{\mathrm{EM}, 1}+\boldsymbol{\Gamma}^{\mathrm{EM}, e} & \boldsymbol{\Gamma}^{\mathrm{EE}, e} & \boldsymbol{\Gamma}^{\mathrm{EM}, e} \\
\boldsymbol{\Gamma}^{\mathrm{ME}, 1}+\Gamma^{\mathrm{ME}, e} & \boldsymbol{\Gamma}^{\mathrm{MM}, 1}+\boldsymbol{\Gamma}^{\mathrm{MM}, e} & \boldsymbol{\Gamma}^{\mathrm{ME}, e} & \boldsymbol{\Gamma}^{\mathrm{MM}, e} \\
\boldsymbol{\Gamma}^{\mathrm{EE}, e} & \boldsymbol{\Gamma}^{\mathrm{EM}, e} & \boldsymbol{\Gamma}^{\mathrm{EE}, 2}+\boldsymbol{\Gamma}^{\mathrm{EE}, e} & \boldsymbol{\Gamma}^{\mathrm{EM}, 2}+\boldsymbol{\Gamma}^{\mathrm{EM}, e} \\
\boldsymbol{\Gamma}^{\mathrm{ME}, e} & \boldsymbol{\Gamma}^{\mathrm{MM}, e} & \boldsymbol{\Gamma}^{\mathrm{ME}, 2}+\boldsymbol{\Gamma}^{\mathrm{ME}, e} & \boldsymbol{\Gamma}^{\mathrm{MM}, 2}+\boldsymbol{\Gamma}^{\mathrm{MM}, e}
\end{array}\right) .
$$

Note that the quantity $\boldsymbol{\Gamma} \cdot\left(\begin{array}{c}\mathbf{K} \\ \mathbf{N}\end{array}\right)$ in (43) is nothing but the lefthand side of Eq. (B13).

Having elucidated the structure of the effective action for surface currents, the remainder of our derivation is now straightforward. To evaluate the functional integral over sources in (43), we approximate $\mathbf{K}$ and $\mathbf{N}$ as expansions in a finite set of tangential basis functions representing surface currents flowing on the surface of the interacting Casimir objects, as is done in the boundary-element method of computational electromagnetism [cf. Eq. (B12)]:

$$
\mathbf{K}(\mathbf{x})=\sum k_{\alpha} \mathbf{f}_{\alpha}(\mathbf{x}), \quad \mathbf{N}(\mathbf{x})=-\sum n_{\alpha} \mathbf{f}_{\alpha}(\mathbf{x}) .
$$

We now insert these expansions into (43) and approximate the infinite-dimensional integrals over $\mathbf{K}$ and $\mathbf{N}$ as finitedimensional integrals over the $\mathbf{k}_{\alpha}$ and $\mathbf{n}_{\alpha}$ coefficients:

$$
\int \mathcal{D} \mathbf{K}_{i} \mathcal{D} \mathbf{N}_{i} \Longrightarrow \mathcal{J} \int \prod_{\alpha} d k_{\alpha} \prod_{\alpha} d n_{\alpha},
$$

where $\mathcal{J}$, the Jacobian of the variable transformation, is an unimportant constant that cancels upon taking the ratio in (18) (and which is not written out in the equations below).
Equation (43) now becomes simply

$$
\mathcal{Z}(\beta, \xi)=\int \prod_{\alpha} d k_{\alpha} \prod_{\alpha} d n_{\alpha} e^{-\frac{\xi}{2 \beta}\left(\begin{array}{l}
\mathrm{k} \\
\mathbf{k}
\end{array}\right)^{\mathrm{T}} \cdot \mathbf{M}(\xi) \cdot\left(\begin{array}{l}
\mathrm{k} \\
\mathrm{n}
\end{array}\right)},
$$

a finite-dimensional Gaussian integral which we evaluate immediately to obtain

$$
=\{\#\} \cdot[\operatorname{det} \mathbf{M}(\xi)]^{-1 / 2},
$$

where $\mathbf{M}(\xi)$ is nothing but the SIE matrix discussed in Appendix B (and, once again, $\{\#\}$ is just an irrelevant constant into which the $\xi / 2 \beta$ prefactor in the exponent disappears). Now finally inserting (44) into (19) and (18) leads immediately back to our FSC formulas (1), and our derivation is complete.

\section{EQUALITY OF THE PARTIAL TRACES}

An important practical simplification of the FSC formulas follows from the structure of the BEM matrices. Recall from (1) that the quantity that enters into the FSC expression 
for the Casimir force is

$$
\operatorname{Tr}\left\{\mathbf{M}^{-1} \cdot \frac{\partial \mathbf{M}}{\partial \mathbf{r}_{i}}\right\}=\sum_{\alpha \beta} \mathbf{M}_{\alpha \beta}^{-1}\left[\frac{\partial \mathbf{M}_{\beta \alpha}}{\partial \mathbf{r}_{i}}\right]
$$

(with a similar expression for the torque). We show that the trace in (45) neatly splits into two equal subtraces, and thus that to compute the full trace we need only sum a subset of the diagonal elements of the matrix in curly brackets (and double the result). In practice this reduces the computational expense of the trace computation by a factor of two or greater.

To understand the physical intuition behind this simplification, note that the sum in (45) runs over the elements of our basis of surface-current expansion functions, which includes functions defined on the surfaces of each of the objects in our Casimir geometry. In evaluating the portion of this sum contributed by basis functions defined on a single object surface, we are, in effect, computing a sort of surface integral over the surface of that object. Intuitively, we might expect that, to compute the force on one object, it would suffice to evaluate this surface integral over the surface of that object alone, or over the surfaces of all other objects, but that we need not do both. As we now show, this physical expectation is borne out by the mathematics; to compute the full trace in (45) we need only sum the contributions of basis functions on the surface of the object on which we are computing the force and double the result.

As before, let the objects in our Casimir geometry be labeled $\mathcal{O}_{r}, r=1,2, \ldots$, with $\mathcal{O}_{1}$ the object on which we are computing the Casimir force, and let $D_{r}$ be the dimension of the subblock of the $\mathbf{M}$ matrix corresponding to object $\mathcal{O}_{r}$ (that is, $D_{r}$ is the number of surface-current basis functions defined on the surface of $\mathcal{O}_{r}$ ). The dimension of the full matrix is $D=\sum D_{r}$.

A rigid displacement of object $\mathcal{O}_{1}$ leaves unchanged the interactions between all pairs of basis functions save those pairs in which precisely one basis function lives on $\mathcal{O}_{1}$. We can thus split the sum in (45) into two pieces:

$$
\begin{aligned}
\sum_{\alpha \beta} M_{\alpha \beta}^{-1}\left[\frac{\partial M_{\alpha \beta}}{\partial \mathbf{r}_{i}}\right]= & \sum_{\alpha=1}^{D_{1}} \sum_{\beta=D_{1}+1}^{D} M_{\alpha \beta}^{-1}\left[\frac{\partial M_{\beta \alpha}}{\partial \mathbf{r}_{i}}\right] \\
& +\sum_{\alpha=D_{1}+1}^{D} \sum_{\beta=1}^{D_{1}} M_{\alpha \beta}^{-1}\left[\frac{\partial M_{\beta \alpha}}{\partial \mathbf{r}_{i}}\right] .
\end{aligned}
$$

The first piece on the right-hand side here is the sum of the first $D_{1}$ diagonal elements of the matrix $\mathbf{M}^{-1} \cdot \frac{\partial \mathbf{M}}{d \mathbf{r}_{i}}$, while the second piece is the sum of the remaining $D-D_{1}$ elements. However, from the fact that $\mathbf{M}$ is a symmetric matrix $\left(M_{\alpha \beta}=M_{\beta \alpha}\right)$ it now follows that the two pieces here are equal, and thus to compute the full trace we need only sum the first $D_{1}$ diagonal elements of $\mathbf{M}^{-1} \cdot \frac{\partial \mathbf{M}}{d \mathbf{r}_{i}}$ (or the latter $D-D_{1}$ elements, if they are fewer) and double the result; i.e.,

$$
\operatorname{Tr} \mathbf{M}^{-1} \cdot \frac{\partial \mathbf{M}}{\partial \mathbf{r}_{\mathrm{i}}}=2 \sum_{\alpha=1}^{\mathrm{D}_{1}} \sum_{\beta=\mathrm{D}_{1}+1}^{\mathrm{D}} \mathbf{M}_{\alpha \beta}^{-1}\left[\frac{\partial \mathbf{M}_{\alpha \beta}}{\partial \mathbf{r}_{\mathrm{i}}}\right]
$$

In practice, a convenient way to evaluate this quantity is to $\mathrm{LU}$ factorize the matrix $\mathbf{M}$, solve the linear systems $\mathbf{M} \cdot \mathbf{X}_{m}=\mathbf{B}_{m}$, where the vectors $\mathbf{X}_{m}$ are the first $D_{1}$ columns of $\frac{\partial \mathbf{M}}{\partial \mathbf{r}_{i}}$, then extract and sum the $m$ th elements of the vectors $\mathbf{B}_{m}$ and double the result. The equality of the partial traces then ensures that this operation requires just $D_{1}$ linear solves, in contrast to the full $D$ solves that would be required in the absence of the simplification.

\section{APPLICATIONS}

\section{A. Casimir forces between metallic spheres and cubes}

To validate our method and demonstrate its flexibility, we first calculate the Casimir force between pairs of metallic particles of spherical and cubical shapes (Fig. 2).

For the sphere-sphere case, the Casimir force may be computed using scattering-matrix methods based on a sphericalwave decomposition of the electromagnetic field [44,45], with the Casimir energy expressed in terms of interactions among waves labeled by the usual spherical indices $\{\ell, m\}$. At large values of the sphere-sphere separation, only waves with small values of $\ell$ are relevant, and in this regime Ref. [44] obtained an asymptotic power series for the force, whose first four terms we have plotted in Fig. 2 (lower dashed curve). At smaller values of the sphere-sphere separation, the sum over spherical waves may be evaluated numerically, as is done in Fig. 2 (open blue circles) with waves up to $\ell=40$ retained for each of the two possible polarizations, corresponding to $2(\ell+1)^{2}=3362$ basis functions for each sphere.

To perform the calculation using FSC techniques, we discretize the surfaces of the objects into small surface patches and expand surface currents using the localized basis functions

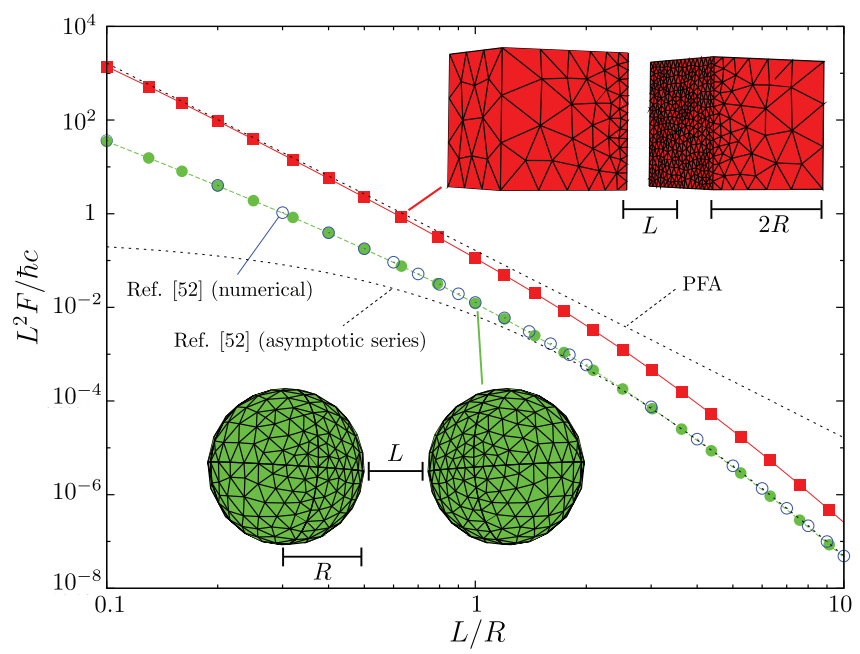

FIG. 2. (Color online) Casimir force between PEC spheres and between PEC cubes. The solid green circles indicate sphere-sphere data computed using the FSC method described in this paper, while the open blue circles indicate sphere-sphere data computed using a numerical implementation of the scattering-matrix method of Ref. [44]. (The lower dashed-line curve indicates the first four terms of the asymptotic series for the sphere-sphere Casimir force reported in Ref. [44].) The solid red squares indicate cube-cube data computed using the FSC method described in this paper; for this geometry, scattering-matrix methods and indeed almost all existing Casimir methods would be unwieldy or impossible to apply. The upper dashed-line curve indicates the proximity-force approximation (PFA) to the cube-cube force, $F_{\mathrm{PFA}}=4 R^{2} \frac{\pi^{2} \hbar c}{240 L^{4}}$ for cubes of face area $4 R^{2}$. 
of Fig. 1; in this case, the number of basis functions retained in the description of the surface currents is 2976 for each sphere. As illustrated in Fig. 2, the FSC calculation (solid red circles) reproduces the results of the spherical-wave calculation. Note that our choice of surface-mesh basis functions allows us to concentrate more degrees of freedom in the regions where we expect the surface source densities to be most rapidly varying-namely, the regions of each sphere that most closely approach the other sphere-while simultaneously using a relatively coarse-grained representation of weakly interacting regions. Thus, already for the simple sphere-sphere geometry our method begins to exhibit practical advantages over spherical-wave-basis methods, in which the resolution can be increased only globally rather than locally.

The FSC method really comes into its own in treating objects that cannot be efficiently described by analytical Maxwell solutions, such as the case of two cubes. To handle such a geometry using scattering-matrix methods, we would be forced either to expand fields in and around the cubes in spherical waves - an approach which would require retaining basis functions up to inordinately large values of $\ell$ except in the long-distance limit [46]—or to reformulate the method in a basis of Maxwell solutions for cubical scatterers, for which analytical expressions are not available. In contrast, the FSC approach handles the geometry with no more effort than is required for the sphere-sphere case (inset and solid blue circles in Fig. 2).

An additional limitation of the spherical-wave approach is that it is inherently restricted to separation distances large enough that the interacting objects may be enclosed in nontouching, nonoverlapping spheres. For the cube-cube geometry pictured in Fig. (2), this would render the method inapplicable for distances $L / R<1.47$, excluding much of the range plotted in the figure.

\section{B. Repulsion of an anisotropic nanoparticle from a square aperture in a thin metallic plate}

Reference [6] considered the Casimir force on an elongated nanoparticle above an aperture in a thin metallic plate and predicted a regime in which the force on the nanoparticle is repulsive. This work, as well as subsequent investigations of similar phenomena [47,48], considered only circular apertures; here we investigate the case of a square aperture (Fig. 3). We consider the Casimir force on a cylindrical nanoparticle (lower inset), whose axis coincides with the axis of a thin $(20-\mathrm{nm})$ plate with a $1-\mu \mathrm{m}$ square aperture, as a function of the distance $Z$ between the vertical center of the nanoparticle and the center of the thin plate. Both cylinder and plate are made of real (lossy) gold, described by a relative dielectric function $\epsilon(\xi)=1+w_{p}^{2} /[\xi(\xi+\gamma)]$ with $\left\{w_{p}, \gamma\right\}=\left\{1.37 \times 10^{16}, 5.32 \times 10^{13}\right\} \mathrm{rad} / \mathrm{s}$. When the center of the nanoparticle is vertically aligned with the center of the plate $(Z=0)$, the $z$-directed Casimir force vanishes by symmetry; as the nanoparticle is displaced slightly in the positive $z$ direction it experiences first a repulsive Casimir force (shaded region of plot) which peaks near the value of $Z$ at which the nanoparticle first lies entirely above the plate. As the nanoparticle is raised further above the plate, the repulsive force decreases in magnitude and eventually crosses

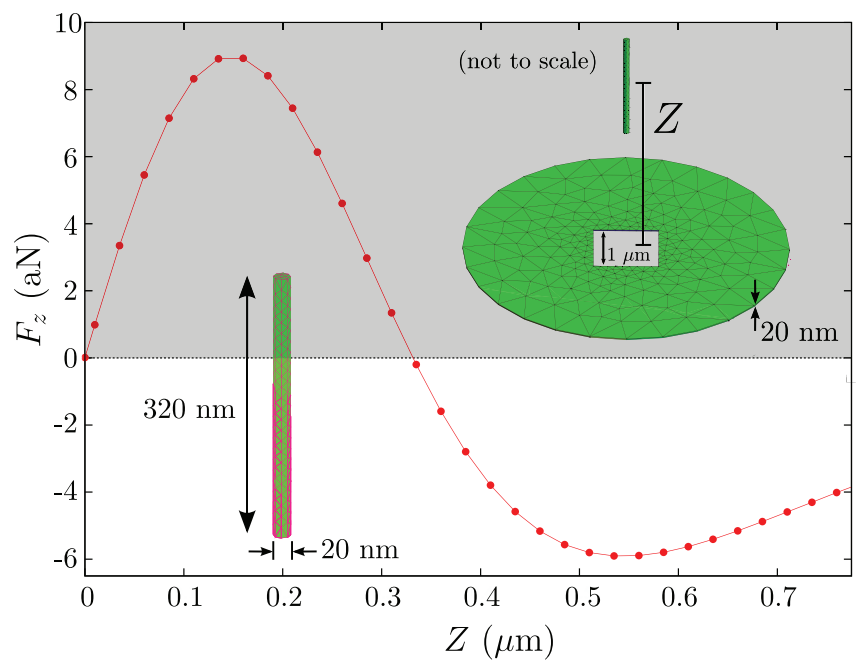

FIG. 3. (Color online) Casimir force on an elongated cylindrical nanoparticle above a square aperture in a thin plate. The upper inset (not to scale) shows the particle-plate geometry, while the lower inset is a close-up view of the surface mesh used to represent the particle. The axis of the nanoparticle (the $z$ axis) is perpendicular to the plane of the plate; the center of the nanoparticle lies a distance $Z$ above the center of the plate. In the regime $0 \leqslant Z \leqslant 330 \mathrm{~nm}$, the $z$-directed force on the nanoparticle is positive; i.e., the particle is repelled from the plate. The shaded portion of the graph indicates this repulsive-force regime. For $Z>185$ the nanoparticle lies entirely above the plate and the force is thus unambiguously repulsive. Both the nanoparticle and the plate are made of finite-conductivity gold (see text).

over into an attractive force whose magnitude decays at large $Z$ (unshaded region of the plot).

The fact that the square-hole geometry reproduces the repulsion phenomenon observed in the circular-hole case is not particularly surprising, but we note that this geometry exhibits several features which would make it prohibitively expensive if not outright impossible to treat using any other Casimir method of which we are aware. In particular, the interpenetrating nature of the nanoparticle-plate configuration would immediately render most scattering-matrix methods inapplicable. Moreover, the drastic distance in length scales between the tiny (20-nm-diameter) cylinder and the relatively large (5- $\mu \mathrm{m}$-radius) plate, together with the absence of rotational symmetry, would pose severe challenges to finite-difference methods. In contrast, the FSC method easily accommodates nonuniform surface meshes, allowing us to describe the nanoparticle surface as a union of appropriately sized surface patches even as we use much larger patches for the plate surface.

\section{CONCLUSIONS}

The FSC approach to Casimir physics, together with other recently developed Casimir methods such as the finitedifference approaches [30,31,49,50], constitutes an advance in the development of Casimir algorithms that mirrors an earlier evolution in computational electromagnetism (EM). In the latter field, traditional special-function approaches such as Mie's method for spherical scattering began to be 
complemented in the 1960 s and 1970 s by a host of numerical techniques that expanded the range of geometries that could be accurately and efficiently treated. Today, numerical techniques for general geometries coexist with geometry-specific specialfunction approaches to constitute a rich and varied arsenal of computational EM techniques appropriate for almost any conceivable situation.

In the future, we expect a similar situation to prevail in the Casimir field. General-purpose methods such as the one presented here will not replace scattering-matrix Casimir methods any more than finite-difference Maxwell solvers have replaced the theory of Mie scattering; instead, methods such as our FSC technique will coexist with scatteringmatrix methods, augmenting the toolbox of available methods available for predicting Casimir interactions across the gamut of geometric and material configurations.

Among general-geometry numerical Casimir methods, the FSC approach is unique in obtaining compact determinant and trace formulas for Casimir quantities. All other generalpurpose numerical methods rely on numerical evaluation of a surface integral for the Casimir force and torque (and on even more unwieldy numerical volume integrations for the Casimir energy). For this reason, we expect the FSC approach to be the most efficient numerical Casimir method for the piecewise-homogeneous material configurations to which it applies.

In addition to the practical usefulness of the FSC Casimir formulas, the two independent derivations that we have provided in this paper contain a number of theoretical innovations that we expect to find broader application. In particular, in the stress-tensor derivation we stated and proved a new integral identity involving the homogeneous DGFs of Maxwell's equations (Appendix C), while in the path-integral derivation we introduced a new type of Lagrange multiplier to constrain the functional integration over the photon field. We hope the latter technique will prove to be a generally useful tool in quantum field theory; one possible application beyond the Casimir realm is the boundary dependence of entanglement entropy in the electromagnetic field, a subject recently addressed for scalar fields [51].

What challenges lie ahead for FSC Casimir computations? If the evolution of computational Casimir physics continues to mimic that of computational EM, an obvious next step will be the development of fast solvers [52]—algorithms that exploit physical insight to reduce the computational complexity of matrix manipulations in equations like (1) from $O\left(N^{3}\right)$ to a more tractable scaling such as $O(N \ln N)$, where $N$, the dimension of the matrix, is the number of surface-current expansion functions $\left\{\mathbf{f}_{\alpha}\right\}$ retained in Eqs. (B3) and (B12). Although a number of algorithms are known for evaluating matrix-vector products involving SIE matrices in $O(N \ln N)$ time [52], the question of how best to exploit such algorithms to evaluate the determinant and trace in Eqs. (1) is nontrivial. To date, all FSC Casimir calculations have considered problems of moderate complexity $\left(N \lesssim 10^{4}\right)$, for which dense-direct linear-algebra solvers are adequate; however, future problems may require going beyond this regime, in which case fast solvers will be essential.

A separate challenge is to apply FSC techniques to the calculation of Casimir forces out of thermal equilibrium, as well as to the closely related problem of near-field radiative heat transfer. These problems, which represent a logical next step in the study of fluctuation-induced forces beyond the equilibrium Casimir case, have begun very recently to be investigated using scattering-matrix $[26,53,54]$ and numerical [55] methods. A hybrid technique combining scattering-matrix ideas with SIE-based numerical calculations was proposed in [8], but was restricted to a cylindrical-wave basis; to date there has been no basis-independent surface-current formulation of nonequilibrium fluctuation problems. Could the FSC technique presented in this paper be modified to apply to these problems? If so, what physical insight would the surface-current formulation lend, and what improvements could be achieved in the efficiency of practical calculations?

In this paper we have said almost nothing about the practical challenges inherent in concrete numerical implementations of the FSC formulas. Among these are (a) how best to choose the surface-current basis functions $\left\{\mathbf{f}_{\alpha}\right\}$ for a given geometry, (b) how to evaluate the multidimensional integrals that enter into the elements of the $\mathbf{M}$ matrix [such as Eqs. (B6)]; (c) how to compute the matrix determinant, inverse, and trace in Eqs. (1), and (d) how to evaluate the imaginary-frequency integrations in Eqs. (1), as well as the Matsubara sums in their finite-temperature analogs. All of these challenges will be addressed in subsequent publications.

\section{ACKNOWLEDGMENTS}

We are grateful to Alejandro Rodriguez for providing the scattering-matrix sphere-sphere data of Fig. 2. The authors are grateful for support from the Singapore-MIT Alliance Computational Engineering flagship research program. This work was supported in part by the Defense Advanced Research Projects Agency (DARPA) under Grant No. N66001-09-1-2070-DOD, by the Army Research Office through the Institute for Soldier Nanotechnologies (ISN) under Grant No. W911NF-07-D0004, and by the AFOSR Multidisciplinary Research Program of the University Research Initiative (MURI) for Complex and Robust On-chip Nanophotonics under Grant No. FA9550-091-0704.

\section{APPENDIX A: HOMOGENEOUS AND SCATTERING DYADIC GREEN'S FUNCTIONS}

For reference, we collect here some well-known results concerning the DGFs of classical electromagnetic theory [56,57].

In the presence of known volume densities of electric and magnetic current $\mathbf{J}(\mathbf{x}), \mathbf{M}(\mathbf{x})$ at a fixed imaginary frequency $\xi$, the components of the electric and magnetic fields are given by linear convolution relations of the form

$$
\begin{aligned}
& E_{i}(\mathbf{x})=\int\left\{\Gamma_{i j}^{\mathrm{EE}}\left(\xi ; \mathbf{x}, \mathbf{x}^{\prime}\right) J_{j}\left(\mathbf{x}^{\prime}\right)+\Gamma_{i j}^{\mathrm{EM}}\left(\xi ; \mathbf{x}, \mathbf{x}^{\prime}\right) M_{j}\left(\mathbf{x}^{\prime}\right)\right\} d \mathbf{x}^{\prime}, \\
& H_{i}(\mathbf{x})=\int\left\{\Gamma_{i j}^{\mathrm{ME}}\left(\xi ; \mathbf{x}, \mathbf{x}^{\prime}\right) J_{j}\left(\mathbf{x}^{\prime}\right)+\Gamma_{i j}^{\mathrm{MM}}\left(\xi ; \mathbf{x}, \mathbf{x}^{\prime}\right) M_{j}\left(\mathbf{x}^{\prime}\right)\right\} d \mathbf{x}^{\prime} .
\end{aligned}
$$

These relations define the four DGFs $\boldsymbol{\Gamma}$.

In an infinite homogeneous medium with spatially constant relative permeability and permittivity $\epsilon(\xi ; \mathbf{x})=\epsilon^{r}(\xi)$, $\mu(\xi ; \mathbf{x})=\mu^{r}(\xi)$, the four $\boldsymbol{\Gamma}$ functions may be expressed in 
terms of just two tensors:

$$
\begin{aligned}
\Gamma^{\mathrm{EE}, r}\left(\xi, \mathbf{r}, \mathbf{r}^{\prime}\right) & =-Z_{0} Z^{r} \kappa^{r} \mathbf{G}\left(\kappa^{r}, \mathbf{r}-\mathbf{r}^{\prime}\right), \\
\Gamma^{\mathrm{ME}, r}\left(\xi, \mathbf{r}, \mathbf{r}^{\prime}\right) & =\kappa^{r} \mathbf{C}\left(\kappa^{r}, \mathbf{r}-\mathbf{r}^{\prime}\right), \\
\Gamma^{\mathrm{EM}, r}\left(\xi, \mathbf{r}, \mathbf{r}^{\prime}\right) & =-\kappa^{r} \mathbf{C}\left(\kappa^{r}, \mathbf{r}-\mathbf{r}^{\prime}\right), \\
\boldsymbol{\Gamma}^{\mathrm{MM}, r}\left(\xi, \mathbf{r}, \mathbf{r}^{\prime}\right) & =-\frac{\kappa^{r}}{Z_{0} Z^{r}} \mathbf{G}\left(\kappa^{r}, \mathbf{r}-\mathbf{r}^{\prime}\right), \\
\left(Z_{0}=\sqrt{\frac{\mu_{0}}{\epsilon_{0}}}, \quad Z^{r}\right. & \left.=\sqrt{\frac{\mu^{r}}{\epsilon^{r}}}, \quad \kappa^{r}=\sqrt{\mu_{0} \mu^{r} \epsilon_{0} \epsilon^{r}} \xi\right),
\end{aligned}
$$

where G, sometimes referred to as the "photon Green's function," is the solution to the equation

$$
\left[\nabla \times \nabla \times+\kappa^{2}\right] \mathbf{G}(\kappa ; \mathbf{r})=\delta(\mathbf{r}) \mathbf{1}
$$

and $\mathbf{C}$ is defined by

$$
\mathbf{C}=\frac{1}{\kappa} \nabla \times \mathbf{G}
$$

(Note that $\mathbf{G}$ and $\mathbf{C}$ have dimensions of inverse length, while the $\boldsymbol{\Gamma}$ functions have dimensions of field or surface current density; for example, $\Gamma^{\mathrm{ME}}$ has dimensions of magnetic field or electric surface current density.)

Explicit expressions for the components of $\mathbf{G}$ and $\mathbf{C}$ are

$$
G_{i j}=\left[\delta_{i j}-\frac{1}{\kappa^{2}} \partial_{i} \partial_{j}\right] G_{0}, \quad C_{i j}=-\frac{1}{\kappa} \varepsilon_{i j k} \partial_{k} G_{0},
$$

where $G_{0}$ is the scalar Green's function for the Helmholtz equation,

$$
G_{0}\left(\kappa ; \mathbf{r}-\mathbf{r}^{\prime}\right)=\frac{e^{-\kappa\left|\mathbf{r}-\mathbf{r}^{\prime}\right|}}{4 \pi\left|\mathbf{r}-\mathbf{r}^{\prime}\right|},
$$

which satisfies

$$
\left[\nabla^{2}-\kappa^{2}\right] G_{0}\left(\kappa ; \mathbf{r}-\mathbf{r}^{\prime}\right)=\delta\left(\mathbf{r}-\mathbf{r}^{\prime}\right) .
$$

\section{Momentum-space representations}

The momentum-space decomposition of the scalar Green's function (A5) is

$$
G_{0}(\kappa ; \mathbf{r})=\int \frac{d \mathbf{k}}{(2 \pi)^{3}} \tilde{G}_{0}(\kappa ; \mathbf{k}) e^{i \mathbf{k} \cdot \mathbf{r}},
$$

with

$$
\tilde{G}_{0}(\kappa ; \mathbf{k})=\frac{1}{\kappa^{2}+|\mathbf{k}|^{2}} .
$$

(In what follows we generally omit the $\sim$ designation, relying on context to differentiate between real- and momentum-space functions.)

The momentum-space version of (A4) reads

$$
G_{i j}=\left[\delta_{i j}+\frac{k_{i} k_{j}}{\kappa^{2}}\right] G_{0}, \quad C_{i j}=-\frac{i}{\kappa} \varepsilon_{i j k} k_{k} G_{0},
$$

or, in matrix format,

$$
\begin{aligned}
\mathbf{G}(\mathbf{k})= & \frac{1}{\kappa^{2}\left(\kappa^{2}+|\mathbf{k}|^{2}\right)}\left[\left(\begin{array}{ccc}
\kappa^{2} & 0 & 0 \\
0 & \kappa^{2} & 0 \\
0 & 0 & \kappa^{2}
\end{array}\right)\right. \\
& \left.+\left(\begin{array}{ccc}
k_{x}^{2} & k_{x} k_{y} & k_{x} k_{z} \\
k_{y} k_{x} & k_{y}^{2} & k_{y} k_{z} \\
k_{z} k_{x} & k_{z} k_{y} & k_{z}^{2}
\end{array}\right)\right] \\
\mathbf{C}(\mathbf{k})= & \frac{i}{\kappa\left(\kappa^{2}+|\mathbf{k}|^{2}\right)}\left(\begin{array}{ccc}
0 & -k_{z} & k_{y} \\
k_{z} & 0 & -k_{x} \\
-k_{y} & k_{x} & 0
\end{array}\right) .
\end{aligned}
$$

\section{Scattering dyadic Green's functions}

In a general inhomogeneous region, the DGFs may be expressed as the sum of two terms,

$$
\Gamma^{\mathrm{EE}}\left(\xi ; \mathbf{x}, \mathbf{x}^{\prime}\right)=\Gamma^{\mathrm{EE}, \mathbf{x}}\left(\xi ; \mathbf{x}-\mathbf{x}^{\prime}\right)+\mathcal{G}^{\mathrm{EE}}\left(\xi ; \mathbf{x}, \mathbf{x}^{\prime}\right)
$$

(and similarly for the other three $\boldsymbol{\Gamma}$ functions); here $\Gamma^{\mathrm{EE}, \mathbf{x}}$ is the homogeneous DGF for an infinite medium with constant $\epsilon^{r}, \mu^{r}$ set equal to their values at $\mathbf{x}$, and $\mathcal{G}^{\mathrm{EE}}$ is the scattering part of the DGF, which describes the fields scattered from the inhomogeneities in the geometry. The first term in (A8) is singular as $\mathbf{x} \rightarrow \mathbf{x}^{\prime}$, but the second term is perfectly well defined in that limit and is the quantity that enters into the fluctuation-dissipation expressions for the spectral density of fluctuations in products of field components, as discussed in Sec. II.

\section{APPENDIX B: A REVIEW OF THE SURFACE-INTEGRAL-EQUATION FORMULATION OF CLASSICAL ELECTROMAGNETISM}

Computational Casimir physics is intimately related to the theory of classical electromagnetic scattering, and many practical methods for predicting Casimir interactions are based on well-known techniques for solving scattering problems. Among the classical scattering methods that have been appropriated for Casimir purposes are the $T$-matrix method [2,24, 58], the method of reflection coefficients [23], and the numerical finite-difference method [30,31]. The method discussed in this paper derives from yet another well-known approach to scattering problems, namely, the method of SIEs. (SIE techniques were first used for Casimir studies in Ref. [4], while Refs. [32,37] presented an SIE-based implementation of the numerical stress-tensor method.) As background for the main text of this paper, in this appendix we review the well-known SIE procedure.

Surface-integral techniques have a long history in electromagnetic theory [59], dating back to the equivalence principles of Love [60] and Schelkunoff [61] and the Stratton-Chu equations [62] from the first half of the 20th century. Numerical implementation of SIEs-known as the "boundary-element method" (BEM) or the "method of moments"- - emerged in the 1970 s as an alternative to other computational procedures such as the finite-difference method (FDM) and the finite-element method (FEM) [57,63]. Whereas the FDM and the FEM 


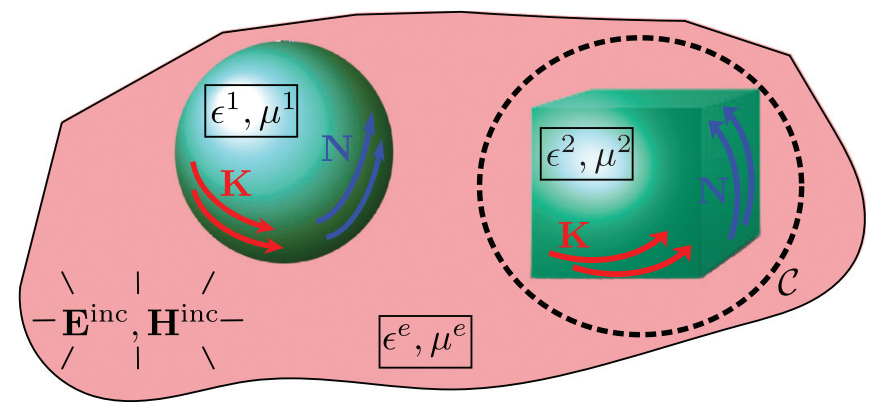

FIG. 4. (Color online) Schematic depiction of a scattering geometry in the SIE picture. A collection of arbitrarily shaped homogeneous bodies, with frequency-dependent relative electrical properties $\left\{\epsilon^{r}, \mu^{r}\right\}$, is embedded in a homogeneous medium with electrical properties $\left\{\epsilon^{e}, \mu^{e}\right\}$. Incident radiation, characterized by electric and magnetic fields $\mathbf{E}^{\text {inc }}, \mathbf{H}^{\text {inc }}$, impinges on the objects to induce surface currents; for perfectly conducting objects we have only electric surface currents (K), while for general objects we have equivalent electric and magnetic (N) surface currents. The goal of SIE methods is to solve for the surface-current distributions in terms of the incident fields, after which we can compute the scattered fields anywhere in space from the surface currents. [The dotted line indicates a fictitious bounding contour $\mathcal{C}$ surrounding one of the objects over which we integrate the Maxwell stress tensor to compute the Casimir force on that object (Sec. II).]

proceed by numerically solving a spatially local form of Maxwell's equations, and thus allow treatment of materials with essentially arbitrary spatial variation of the dielectric permittivity and magnetic permeability, the SIE approach takes advantage of the analytically known solutions of Maxwell's equations in homogeneous media and thus in practice is most readily applicable to piecewise-homogeneous material configurations. For this reason, while the FDM and FEM have the advantage of being able to treat a wider class of materials, the SIE method exhibits significant practical advantages for the piecewise-homogeneous geometries typically encountered in Casimir studies.

To fix ideas and notation for the main text of this paper, we here review the SIE formulation of electromagnetic scattering problems, beginning in Appendix B1 with the simplest case of PEC scatterers and then generalizing in Appendix B2 to the case of arbitrary materials.

The material of these two sections is well known and entirely standard within the computational EM literature and is reviewed here only for completeness. However, in Appendix B3 we carry the SIE formalism one step beyond what is usually done to write explicit expressions [Eqs. (B19) and (B22)] for scattering DGFs in terms of the SIE matrices and the homogeneous DGFs.

Throughout this appendix we refer to the scattering situation depicted schematically in Fig. 4, in which a collection of homogeneous scatterers (with frequency-dependent relative electrical properties $\left.\left\{\epsilon^{r}, \mu^{r}\right\}\right)$ is embedded in a homogeneous medium (electrical properties $\left\{\epsilon^{e}, \mu^{e}\right\}$ ) and irradiated by incident radiation characterized by an incident electric field $\mathbf{E}^{\text {inc }}$.

\section{The SIE method For PEC bodies}

We first consider the case in which the scattering objects in Fig. 4 are perfect conductors. An incident field impinging on PEC bodies induces a tangential electric current distribution $\mathbf{K}(\mathbf{x})$ on the body surfaces, which gives rise to a scattered field according to

$$
\mathbf{E}^{\text {scat }}(\mathbf{x})=\int \Gamma^{\mathrm{EE}, e}\left(\mathbf{x}, \mathbf{x}^{\prime}\right) \cdot \mathbf{K}\left(\mathbf{x}^{\prime}\right) d \mathbf{x}^{\prime}
$$

here the integral extends over the surfaces of the bodies and $\Gamma^{\mathrm{EE}, e}$ is the homogeneous DGF for the exterior medium. (Our notation for DGFs is summarized in Appendix A; throughout this section we work at a single frequency and suppress frequency arguments to $\mathbf{E}, \boldsymbol{\Gamma}$, and $\mathbf{K}$.) For a given incident field $\mathbf{E}^{\text {inc }}$ we can solve for $\mathbf{K}$ by requiring that the total (incident + scattered) field satisfy the appropriate boundary condition, which for PEC bodies is simply that the total tangential $\mathbf{E}$ field vanish for all points $\mathbf{x}$ on the body surfaces:

$$
\left[\mathbf{E}^{\text {scat }}(\mathbf{x})+\mathbf{E}^{\text {inc }}(\mathbf{x})\right] \times \hat{\mathbf{n}}(\mathbf{x})=0 .
$$

[Here taking the cross product with $\hat{\mathbf{n}}(\mathbf{x})$, the outward-pointing surface normal at $\mathbf{x}$, is simply a convenient way of extracting the tangential components of a vector.] Inserting (B1) yields an integral equation for $\mathbf{K}(\mathbf{x})$ :

$$
\left[\int \Gamma^{\mathrm{EE}, e}\left(\mathbf{x}, \mathbf{x}^{\prime}\right) \cdot \mathbf{K}\left(\mathbf{x}^{\prime}\right) d \mathbf{x}^{\prime}\right] \times \hat{\mathbf{n}}(\mathbf{x})=-\mathbf{E}^{\mathrm{inc}}(\mathbf{x}) \times \hat{\mathbf{n}}(\mathbf{x}) .
$$

Equation (B2) is known as the "electric-field integral equation (EFIE)" [33].

Thus far all we have done is restate the problem in an integral-equation form. The next step is to discretize this integral equation by introducing a finite set of tangential vector-valued basis functions $\left\{\mathbf{f}_{\alpha}(\mathbf{x})\right\}$, defined on the surfaces of the bodies, which serve a dual purpose as expansion functions for surface currents and test functions for boundary conditions. As noted in Sec. I, an advantage of SIE methods is that they place no restriction on these basis functions; in particular, the $\left\{\mathbf{f}_{\alpha}\right\}$ need not solve the wave equation or any other equation and need not encapsulate any global information
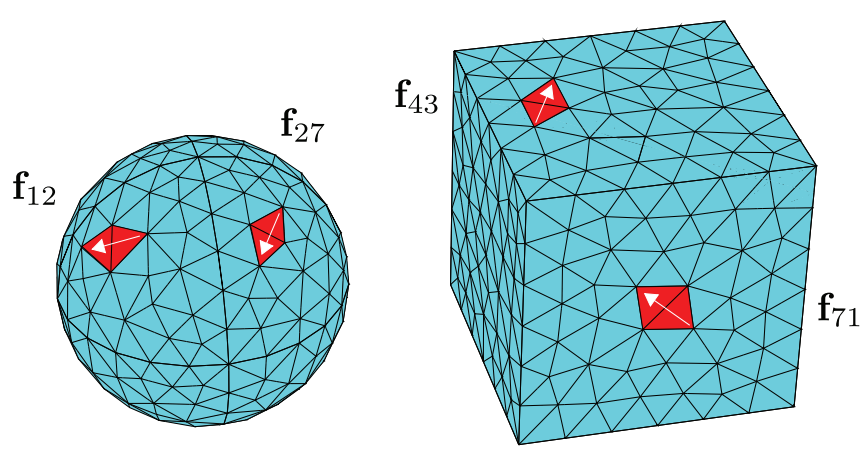

FIG. 5. (Color online) One possible choice of expansion functions for tangential currents on object surfaces is obtained by discretizing object boundaries into unions of small flat panels and introducing localized basis functions describing elemental currents sourced and sunk at panel vertices: These are "RWG" basis functions, associated with each edge (pair of triangles) in the mesh [64]. 
about the scattering geometry. Of course, if symmetries are present, then we may wish to choose the $\left\{\mathbf{f}_{\alpha}\right\}$ in a way that reflects them-we might choose vector spherical harmonics for a spherical scatterer, say, or a Fourier basis for a planar scatterer-but nothing in the SIE formulation requires such a choice, and we are equally free to choose the $\left\{\mathbf{f}_{\alpha}\right\}$ to be arbitrary polynomials, piecewise-linear functions, or any other functions we like. For scatterers of complex geometries, a particularly convenient strategy is to discretize object surfaces into small flat panels and take the $\left\{\mathbf{f}_{\alpha}\right\}$ to describe elemental currents sourced and sunk at panel vertices [64], as depicted in Fig. 5. The localized basis functions that result from such a procedure are known as "boundary elements," and SIE implementations based on them are commonly known as "boundary-element methods' (BEMs) or the "method of moments."

Having chosen a set of basis functions, the surface electric current distribution is approximated as a finite expansion in the $\left\{\mathbf{f}_{\alpha}\right\}$ :

$$
\mathbf{K}(\mathbf{x})=\sum k_{\alpha} \mathbf{f}_{\alpha}(\mathbf{x})
$$

This expansion is then inserted into (B2), and the inner product of that equation is taken with each member in the set $\left\{\mathbf{f}_{\alpha}\right\}$, yielding one equation for each of the unknown coefficients $k_{\alpha}$. Collecting these equations yields a linear system of the form

$$
\mathbf{M k}=\mathbf{v},
$$

where $\mathbf{k}$ is the vector of $k_{\alpha}$ coefficients, the elements of the right-hand-side vector $\mathbf{v}$ describe the interactions of the basis functions with the incident field,

$$
\begin{aligned}
v_{\alpha} & =-\int_{\text {sup } \mathbf{f}_{\alpha}} \mathbf{f}_{\alpha}(\mathbf{x}) \cdot \mathbf{E}^{\text {inc }}(\mathbf{x}) d \mathbf{x} \\
& \equiv-\left\langle\mathbf{f}_{\alpha} \mid \mathbf{E}^{\text {inc }}\right\rangle
\end{aligned}
$$

(where $\sup \mathbf{f}_{\alpha}$ is the support of basis function $\mathbf{f}_{\alpha}$ ), and the elements of the $\mathbf{M}$ matrix describe the interactions of the basis functions with each other through the exterior medium:

$$
\begin{aligned}
\mathbf{M}_{\alpha \beta} & =\int_{\sup \mathbf{f}_{\alpha}} d \mathbf{x} \int_{\sup \mathbf{f}_{\beta}} d \mathbf{x}^{\prime} \mathbf{f}_{\alpha}(\mathbf{x}) \cdot \Gamma^{\mathrm{EE}, e}\left(\mathbf{x}, \mathbf{x}^{\prime}\right) \cdot \mathbf{f}_{\beta}\left(\mathbf{x}^{\prime}\right) \\
& \equiv\left\langle\mathbf{f}_{\alpha}\left|\boldsymbol{\Gamma}^{\mathrm{EE}, e}\right| \mathbf{f}_{\beta}\right\rangle
\end{aligned}
$$

The linear system (B4) may be solved for the surface-current expansion coefficients $\left\{k_{\alpha}\right\}$, after which we can compute the components of the scattered field at an arbitrary point in the exterior medium using the discretized form of Eq. (B1):

$$
\begin{aligned}
E_{i}^{\mathrm{scat}}(\mathbf{x}) & =\sum_{\alpha} k_{\alpha} \int_{\sup _{\alpha} \mathbf{f}_{\alpha}} \Gamma_{i j}^{\mathrm{EE}, e}\left(\mathbf{x}, \mathbf{x}^{\prime}\right) f_{\alpha j}\left(\mathbf{x}^{\prime}\right) d \mathbf{x}^{\prime} \\
& \equiv \sum_{\alpha} k_{\alpha}\left\langle\Gamma_{i}^{\mathrm{EE}, e}(\mathbf{x}) \mid \mathbf{f}_{\alpha}\right\rangle .
\end{aligned}
$$

[The second lines of Eqs. (B5), (B6), and (B7) define some useful shorthand for commonly encountered integrals; in (B7b) note that the contracted index and the integrated argument to $\boldsymbol{\Gamma}$ are suppressed, while the uncontracted index and nonintegrated argument are written out.]

\section{The SIE method for general bodies}

For PEC bodies, the mathematics of the SIE procedure neatly mirrors the physics of the actual situation. Indeed, for good conductors at moderate frequencies it really $i s$ true that the physical induced currents are confined near the object surfaces; the surface current distribution $\mathbf{K}(\mathbf{x})$ for which we solve in an SIE method thus has a direct physical interpretation as an induced surface current.

The situation is more complicated for general (non-PEC) objects, for here the physical induced currents are no longer confined to the surfaces, but instead extend throughout the bulk of the object. The obvious extension of the procedure outlined above would be to introduce a volume discretization and solve a system analogous to (B4) for the coefficients in an expansion of a volume current density $\mathbf{J}(\mathbf{x})$. Such a procedure, while retaining the intuitive interpretation of the quantity computed as a physical current density, would suffer from poor complexity scaling, as the number of unknowns [and thus the dimension of the linear system corresponding to (B4)] would scale like the volume, not the surface area, of the scattering objects.

An alternative approach is to abandon the strategy of solving for the physical volume sources and to solve instead for equivalent surface sources that give rise to the same scattered fields. The mathematical machinery underlying this approach is a vector generalization of Green's theorem known as the Stratton-Chu equations [62], which relate the $\mathbf{E}$ and $\mathbf{H}$ fields in the interior of a region to the tangential components of the fields on the boundary of that region. More precisely, let $\partial \mathcal{O}_{r}$ be the surface of the $r$ th object in our geometry, and for points $\mathbf{x}$ on $\partial \mathcal{O}_{r}$ define two tangential vector fields according to

$$
\mathbf{K}^{\mathrm{eff}}(\mathbf{x}) \equiv \hat{\mathbf{n}}(\mathbf{x}) \times \mathbf{H}(\mathbf{x}), \quad \mathbf{N}^{\mathrm{eff}}(\mathbf{x}) \equiv \mathbf{E}(\mathbf{x}) \times \hat{\mathbf{n}}(\mathbf{x}),
$$

where $\hat{\mathbf{n}}$ is the outward-pointing normal to $\partial \mathcal{O}_{r}$ at $\mathbf{x}$ and where $\mathbf{E}$ and $\mathbf{H}$ are the total fields at that that point. The Stratton-Chu equations are then the following surface-integral expressions for the fields inside and outside $\partial \mathcal{O}_{r}$ :

$$
\begin{gathered}
\mathbf{E}^{\text {in }}=-\int_{\partial \mathcal{O}_{r}}\left\{\boldsymbol{\Gamma}^{\mathrm{EE}, n} \cdot \mathbf{K}^{\mathrm{eff}}+\boldsymbol{\Gamma}^{\mathrm{EM}, n} \cdot \mathbf{N}^{\mathrm{eff}}\right\} d \mathbf{x}^{\prime}, \\
\mathbf{H}^{\mathrm{in}}=-\int_{\partial \mathcal{O}_{r}}\left\{\boldsymbol{\Gamma}^{\mathrm{ME}, n} \cdot \mathbf{K}^{\mathrm{eff}}+\boldsymbol{\Gamma}^{\mathrm{MM}, n} \cdot \mathbf{N}^{\mathrm{eff}}\right\} d \mathbf{x}^{\prime}, \quad(\mathrm{B} 9 \mathrm{a}) \\
\mathbf{E}^{\text {out }}=\mathbf{E}^{\text {inc }}+\int_{\cup \partial \mathcal{O}_{r}}\left\{\boldsymbol{\Gamma}^{\mathrm{EE}, e} \cdot \mathbf{K}^{\mathrm{eff}}+\boldsymbol{\Gamma}^{\mathrm{EM}, e} \cdot \mathbf{N}^{\mathrm{eff}}\right\} d \mathbf{x}^{\prime}, \quad(\mathrm{B} 10 \mathrm{a}) \\
\mathbf{H}^{\text {out }}=\mathbf{H}^{\mathrm{inc}}+\int_{\cup \partial \mathcal{O}_{r}}\left\{\boldsymbol{\Gamma}^{\mathrm{ME}, e} \cdot \mathbf{K}^{\mathrm{eff}}+\boldsymbol{\Gamma}^{\mathrm{MM}, e} \cdot \mathbf{N}^{\mathrm{eff}}\right\} d \mathbf{x}^{\prime} .
\end{gathered}
$$

[In Eqs. (B9) and (B10), the $r$ and $e$ superscripts on $\Gamma$ label the homogeneous DGFs for the medium interior to $\partial \mathcal{O}_{r}$ and the exterior medium, respectively; the spatial arguments to $\mathbf{E}$, $\boldsymbol{\Gamma}, \mathbf{K}$, and $\mathbf{N}$ are as in Eq. (B1), but are suppressed here to save space.]

Note the following differences between expressions (B9) and (B10) for the interior and exterior fields: (a) the surface integrals in the two cases differ in sign, arising from the reversal of direction of the surface normal in (B8); (b) the $\boldsymbol{\Gamma}$ dyadics in (B9) are those for the homogeneous medium interior to 
$\mathcal{O}_{r}$, while in (B10) we instead have those for the the exterior medium; (c) in (B9) we integrate over the single surface $\partial \mathcal{O}_{r}$, while in (B10) the integral is over the union of all object surfaces, $\cup \partial \mathcal{O}_{r}$ (which we may think of as the boundary of the exterior medium, $\cup \partial \mathcal{O}_{r}=\partial \mathcal{O}_{e}$ ); (d) the incident fields contribute to expressions (B9) for the exterior fields, but are absent from expressions (B9) for the interior fields.

Although the tangential vector fields defined by (B8) are simply the components of the $\mathbf{E}$ and $\mathbf{H}$ fields and do not correspond to physical source densities, nonetheless the form of Eqs. (B9) and (B10) suggests interpreting $\mathbf{K}^{\text {eff }}$ and $\mathbf{N}^{\text {eff }}$ as effective electric and magnetic surface current densities, which, if known, would allow computation of the fields anywhere in space, just as knowledge of the physical surface current $\mathbf{K}$ suffices in the PEC case to determine uniquely the full scattered field. To emphasize this analogy, we henceforth drop the "eff" designation from $\mathbf{K}$ and $\mathbf{N}$.

As in the PEC case, the $\mathbf{K}$ and $\mathbf{N}$ distributions are determined by requiring that the total fields satisfy appropriate boundary conditions. For non-PEC bodies these are simply that the tangential components of the total fields be continuous across material boundaries; for a point $\mathbf{x}$ on the surface of a body we have

$$
\begin{aligned}
{\left[\mathbf{E}^{\text {out }}(\mathbf{x})-\mathbf{E}^{\text {in }}(\mathbf{x})\right] \times \hat{\mathbf{n}}(\mathbf{x}) } & =0, \\
{\left[\mathbf{H}^{\text {out }}(\mathbf{x})-\mathbf{H}^{\text {in }}(\mathbf{x})\right] \times \hat{\mathbf{n}}(\mathbf{x}) } & =0 .
\end{aligned}
$$

Inserting (B9) and (B10) into (B11) leads to integral equations for $\mathbf{K}$ and $\mathbf{N}$ that generalize Eq. (B2) for non-PEC bodies. As in the PEC case, the next step is to discretize these integral equations by approximating the electric and magnetic surface currents as expansions in a finite set of tangential vector-valued basis functions defined on the object surfaces,

$$
\mathbf{K}(\mathbf{x})=\sum k_{\alpha} \mathbf{f}_{\alpha}(\mathbf{x}), \quad \mathbf{N}(\mathbf{x})=-\sum n_{\alpha} \mathbf{f}_{\alpha}(\mathbf{x}),
$$

and testing the integral equations obtained from (B11) with each basis function. (The minus sign in the magnetic surfacecurrent expansion is a useful convention that leads to a symmetric linear system [63].) The result of this procedure is a linear system of the same general form as (B4), but now enlarged to exhibit a $2 \times 2$ block structure:

$$
\left(\begin{array}{ll}
\mathbf{M}^{\mathrm{EE}} & \mathbf{M}^{\mathrm{EM}} \\
\mathbf{M}^{\mathrm{ME}} & \mathbf{M}^{\mathrm{MM}}
\end{array}\right)\left(\begin{array}{c}
\mathbf{k} \\
\mathbf{n}
\end{array}\right)=\left(\begin{array}{c}
\mathbf{v}^{\mathrm{E}} \\
\mathbf{v}^{\mathrm{M}}
\end{array}\right) .
$$

In Eq. (B13), the elements of the right-hand-side vector describe the interactions of the basis functions with the incident electric and magnetic fields [compare Eq. (B5)],

$$
\left(\begin{array}{c}
v_{\alpha}^{\mathrm{E}} \\
v_{\alpha}^{\mathrm{M}}
\end{array}\right)=-\left(\begin{array}{c}
\left\langle\mathbf{f}_{\alpha} \mid \mathbf{E}^{\mathrm{inc}}\right\rangle \\
\left\langle\mathbf{f}_{\alpha} \mid \mathbf{H}^{\mathrm{inc}}\right\rangle
\end{array}\right),
$$

while the elements of the $\mathbf{M}$ matrix describe the basis functions interacting with each other both through the exterior medium and through the medium interior to one of the scattering bodies. For example, the elements of the $\mathbf{M}^{\mathrm{EE}}$ block are

$$
M_{\alpha \beta}^{\mathrm{EE}}=\left\langle\mathbf{f}_{\alpha}\left|\boldsymbol{\Gamma}^{\mathrm{EE}, e}+\Gamma^{\mathrm{EE}, r}\right| \mathbf{f}_{\beta}\right\rangle
$$

and similarly for the other blocks. (The $\Gamma^{\mathrm{EE}, r}$ term here is present only if basis functions $\mathbf{f}_{\alpha}$ and $\mathbf{f}_{\beta}$ are defined on the surface of the same object $\mathcal{O}_{r}$, while the $\Gamma^{\mathrm{EE}, e}$ term is present even for basis functions defined on the surfaces of different objects.)

After solving (B13), the scattered fields at an arbitrary point $\mathbf{x}$ are obtained, in analogy to Eq. (B7), from the expansions

$$
\begin{aligned}
E_{i}^{\mathrm{scat}}(\mathbf{x}) & \left.=\sum_{\alpha}\left\{k_{\alpha}\left\langle\boldsymbol{\Gamma}_{i}^{\mathrm{EE}, e}(\mathbf{x}) \mid \mathbf{f}_{\alpha}\right\rangle-n_{\alpha}\left\langle\boldsymbol{\Gamma}_{i}^{\mathrm{EM}, e}(\mathbf{x})\right| \mathbf{f}_{\alpha}\right)\right\}, \\
H_{i}^{\mathrm{scat}}(\mathbf{x}) & =\sum_{\alpha}\left\{k_{\alpha}\left\langle\boldsymbol{\Gamma}_{i}^{\mathrm{ME}, e}(\mathbf{x}) \mid \mathbf{f}_{\alpha}\right\rangle-n_{\alpha}\left\langle\boldsymbol{\Gamma}_{i}^{\mathrm{MM}, e}(\mathbf{x}) \mid \mathbf{f}_{\alpha}\right\rangle\right\} .
\end{aligned}
$$

(These are the scattered fields in the exterior region; the expressions for fields in the interior of object $r$ are similar, but involve the homogeneous DGFs $\Gamma^{\mathrm{PQ}, r}$ for the medium interior to object $r$.)

\section{Explicit SIE expressions for dyadic Green's functions}

The discretized SIE method reviewed in the previous two sections is typically employed as a numerical technique, with the linear systems (B4) and (B13) solved using methods of computational linear algebra and the scattered fields (B7)) and (B16) evaluated numerically. In this paper, in contrast, we use the SIE formalism in a somewhat unusual way, by carrying the analytical development one step further than is commonly done. By exploiting the formal solution of Eqs. (B4) and (B13), we obtain useful expressions for scattering DGFs in terms of the formal inverse of the SIE matrix M. These expressions are be used in Sec. II to derive compact FSC expressions relating Casimir quantities to linear-algebraic manipulations of the $\mathbf{M}$ matrix. Although these final expressions will ultimately be evaluated numerically, the analytical expressions derived in this subsection are an important ingredient in their derivation by stress-tensor methods. (The expressions derived in this section are not needed for the path-integral derivation of the FSC Casimir formulas.)

\section{a. The PEC case}

The scattering DGF $\mathcal{G}_{i j}^{\mathrm{EE}}\left(\mathbf{x}, \mathbf{x}^{\prime}\right)$ is the scattered electric field at $\mathbf{x}$ due to a point electric source at $\mathbf{x}^{\prime}$ (Appendix A); here we need the case in which both $\mathbf{x}$ and $\mathbf{x}^{\prime}$ lie in the exterior medium. To compute this quantity using the SIE technique of Appendix B1, we take the incident field to be the field of a unit-strength $j$-directed point electric current source at a point $\mathbf{x}^{\prime}$ in the exterior medium, which is simply

$$
E_{i}^{\mathrm{inc}}(\mathbf{x})=\Gamma_{i j}^{\mathrm{EE}, e}\left(\mathbf{x}, \mathbf{x}^{\prime}\right) .
$$

Then the elements of the right-hand-side vector in (B4) are

$$
v_{\alpha}=-\left\langle\mathbf{f}_{\alpha} \mid \Gamma_{j}^{\mathrm{EE}, e}\left(\mathbf{x}^{\prime}\right)\right\rangle,
$$

while the coefficients in the expansion of the scattered field may be obtained as the formal solution of (B4),

$$
k_{\alpha}=\sum_{\beta} W_{\alpha \beta} V_{\beta}
$$

(where $\mathbf{W}=\mathbf{M}^{-1}$ is the inverse SIE matrix). Inserting (B18) and (B17) into (B7), the scattered field at $\mathbf{x}-$ which is just the 
scattering DGF we are seeking to compute-is

$$
\mathcal{G}_{i j}^{\mathrm{EE}}\left(\mathbf{x}, \mathbf{x}^{\prime}\right)=-\sum_{\alpha \beta}\left\langle\boldsymbol{\Gamma}_{i}^{\mathrm{EE}, e}(\mathbf{x}) \mid \mathbf{f}_{\alpha}\right\rangle W_{\alpha \beta}\left\langle\mathbf{f}_{\beta} \mid \Gamma_{j}^{\mathrm{EE}, e}\left(\mathbf{x}^{\prime}\right)\right\rangle .
$$

We also need the magnetic-magnetic DGF $\mathcal{G}^{\mathrm{MM}}$, which is the scattered magnetic field due to a point magnetic source. This is obtained in easy analogy to the above by (a) taking the incident field to be the field of a magnetic point source instead of an electric point source, which has the effect of substituting $\Gamma^{\mathrm{EM}}$ for $\Gamma^{\mathrm{EE}}$ in (B17), and (b) computing the scattered magnetic field instead of the scattered electric field, which has the effect of substituting $\Gamma^{\mathrm{ME}}$ for $\Gamma^{\mathrm{EE}}$ in (B1). The result is

$$
\mathcal{G}_{i j}^{\mathrm{MM}}\left(\mathbf{x}, \mathbf{x}^{\prime}\right)=-\sum_{\alpha \beta}\left\langle\boldsymbol{\Gamma}_{i}^{\mathrm{ME}, e}(\mathbf{x}) \mid \mathbf{f}_{\alpha}\right\rangle W_{\alpha \beta}\left\langle\mathbf{f}_{\beta} \mid \boldsymbol{\Gamma}_{j}^{\mathrm{EM}, e}\left(\mathbf{x}^{\prime}\right)\right\rangle
$$

\section{b. The general case}

To obtain explicit expressions for scattering DGFs in general geometries, we mimic the procedure followed above, but now using the general SIE formalism outlined in Appendix B1 instead of the PEC formalism of Appendix B1. To compute $\mathcal{G}^{\mathrm{EE}}$, we again take the incident field to be the field of a unit-strength $j$-directed point electric source at a point $\mathbf{x}^{\prime}$ in the exterior medium, in which case the elements of the right-hand side of Eq. (B13) are

$$
\left(\begin{array}{c}
v_{\alpha}^{\mathrm{E}} \\
v_{\alpha}^{\mathrm{M}}
\end{array}\right)=\left(\begin{array}{c}
\left\langle\mathbf{f}_{\alpha} \mid \boldsymbol{\Gamma}_{j}^{\mathrm{EE}, e}\left(\mathbf{x}^{\prime}\right)\right\rangle \\
\left\langle\mathbf{f}_{\alpha} \mid \boldsymbol{\Gamma}_{j}^{\mathrm{ME}, e}\left(\mathbf{x}^{\prime}\right)\right\rangle
\end{array}\right) .
$$

The expansion coefficients that enter into Eq. (B16) are given, in analogy to Eq. (B18), by the formal solution of (B13):

$$
\left(\begin{array}{c}
k_{\alpha} \\
n_{\alpha}
\end{array}\right)=\sum_{\beta}\left(\begin{array}{cc}
W_{\alpha \beta}^{\mathrm{EE}} & W_{\alpha \beta}^{\mathrm{EM}} \\
W_{\alpha \beta}^{\mathrm{ME}} & W_{\alpha \beta}^{\mathrm{MM}}
\end{array}\right)\left(\begin{array}{c}
V_{\beta}^{\mathrm{E}} \\
V_{\beta}^{\mathrm{M}}
\end{array}\right)
$$

Inserting (B20) and (B21) into (B16), and proceeding similarly for the magnetic-magnetic case, then yields the generalization of Eq. (B19) to non-PEC geometries:

$$
\begin{aligned}
& \mathcal{G}_{i j}^{\mathrm{EE}}\left(\mathbf{x}, \mathbf{x}^{\prime}\right) \\
& =-\sum_{\alpha \beta}\left(\begin{array}{c}
\left\langle\boldsymbol{\Gamma}_{i}^{\mathrm{EE}, e}(\mathbf{x}) \mid \mathbf{f}_{\alpha}\right\rangle \\
-\left\langle\boldsymbol{\Gamma}_{i}^{\mathrm{EM}, e}(\mathbf{x}) \mid \mathbf{f}_{\alpha}\right\rangle
\end{array}\right) \cdot\left(W_{\alpha \beta}\right) \cdot\left(\begin{array}{c}
\left\langle\mathbf{f}_{\beta} \mid \boldsymbol{\Gamma}_{j}^{\mathrm{EE}, e}\left(\mathbf{x}^{\prime}\right)\right\rangle \\
\left\langle\mathbf{f}_{\beta} \mid \boldsymbol{\Gamma}_{j}^{\mathrm{ME}, e}\left(\mathbf{x}^{\prime}\right)\right\rangle
\end{array}\right),
\end{aligned}
$$

$$
\begin{aligned}
& \mathcal{G}_{i j}^{\mathrm{MM}}\left(\mathbf{x}, \mathbf{x}^{\prime}\right) \\
& =-\sum_{\alpha \beta}\left(\begin{array}{c}
\left\langle\boldsymbol{\Gamma}_{i}^{\mathrm{ME}, e}(\mathbf{x}) \mid \mathbf{f}_{\alpha}\right\rangle \\
-\left\langle\boldsymbol{\Gamma}_{i}^{\mathrm{MM}, e}(\mathbf{x}) \mid \mathbf{f}_{\alpha}\right\rangle
\end{array}\right) \cdot\left(W_{\alpha \beta}\right) \cdot\left(\begin{array}{c}
\left\langle\mathbf{f}_{\beta} \mid \boldsymbol{\Gamma}_{j}^{\mathrm{EM}, e}\left(\mathbf{x}^{\prime}\right)\right\rangle \\
\left\langle\mathbf{f}_{\beta} \mid \boldsymbol{\Gamma}_{j}^{\mathrm{MM}, e}\left(\mathbf{x}^{\prime}\right)\right\rangle
\end{array}\right) .
\end{aligned}
$$

Equations (B19) and (B22) are the most important results of this appendix. The crucial property of these expressions is that they present the inhomogeneous Green's function in a fully factorized form in which factors depending on $\mathbf{x}$ are separated from those depending on $\mathbf{x}^{\prime}$. In this sense, Eqs. (B19) are similar to Green's-function expansions for special geometries commonly encountered in the literature, such as spherical-harmonic expansions for spherical geometries or Bessel-function expansions for cylindrical geometries [2,13]; the difference, of course, is that (B19) is applicable to arbitrary geometries, with the geometric information encoded in the $\mathbf{W}$ matrix and the basis functions $\left\{\mathbf{f}_{\alpha}\right\}$.

\section{APPENDIX C: PROOF OF INTEGRAL IDENTITIES}

In this Appendix we state and prove an integral identity that underlies the stress-tensor derivation of the FSC formulae presented in Sec. II. An identity bearing at least a superficial resemblance appears in Eq. A.6 of Ref. [65].

In Sec. II, we introduced a three-index integral kernel $\mathcal{I}$ defined by

$$
\mathcal{I}_{i k l}\left(\mathbf{r}, \mathbf{r}^{\prime}\right)=\kappa^{2} \oint_{\mathcal{C}} V_{A}\left(\mathbf{r}, \mathbf{r}^{\prime}, \mathbf{x}\right) n_{A}(\mathbf{x}) d \mathbf{x}
$$

where the integration is over a closed surface in space (a topological two-sphere) $\mathcal{C}$ and the integrand contains products of factors of DGFs:

$$
V_{A}\left(\mathbf{r}, \mathbf{r}^{\prime}, \mathbf{x}\right)=G_{i k}^{\mathbf{1}} G_{l A}^{\mathbf{2}}-\frac{\delta_{i A}}{2} G_{B k}^{\mathbf{1}} G_{l B}^{\mathbf{2}}-C_{i k}^{\mathbf{1}} C_{l A}^{\mathbf{2}}+\frac{\delta_{i A}}{2} C_{B k}^{\mathbf{1}} C_{l B}^{\mathbf{2}} .
$$

Here the $\mathbf{G}$ and $\mathbf{C}$ dyadics are those defined by Eqs. (A2) and (A3); we use capital Roman letters $(A, B, \ldots)$ to denote contracted indices, and we are using a shorthand notation in which $\kappa$ arguments are suppressed and spatial arguments are replaced with superscripts,

$$
G_{i j}^{\mathbf{1}} \equiv G_{i j}(\kappa, \mathbf{x}-\mathbf{r}), \quad G_{i j}^{\mathbf{2}} \equiv G_{i j}\left(\kappa, \mathbf{r}^{\prime}-\mathbf{x}\right) .
$$

We also defined a symmetrized version of $\mathcal{I}$,

$$
\begin{aligned}
\overline{\mathcal{I}}_{i k l}\left(\mathbf{r}, \mathbf{r}^{\prime}\right) & =\mathcal{I}_{i k l}\left(\mathbf{r}, \mathbf{r}^{\prime}\right)+\mathcal{I}_{i l k}\left(\mathbf{r}^{\prime}, \mathbf{r}\right) \\
& \equiv \kappa^{2} \oint_{\mathcal{C}} \bar{V}_{A}(\mathbf{x}) n_{A}(\mathbf{x}) d \mathbf{x}
\end{aligned}
$$

with

$$
\begin{aligned}
\bar{V}_{A}(\mathbf{x})= & G_{i k}^{\mathbf{1}} G_{l A}^{2}+G_{k A}^{\mathbf{1}} G_{i l}^{\mathbf{2}}-\delta_{i A} G_{B k}^{\mathbf{1}} G_{l B}^{2}-C_{i k}^{\mathbf{1}} C_{l A}^{\mathbf{2}} \\
& -C_{k A}^{\mathbf{1}} C_{i l}^{\mathbf{2}}+\delta_{i A} C_{B k}^{\mathbf{1}} C_{l B}^{\mathbf{2}} .
\end{aligned}
$$

The goal of this appendix is to demonstrate that, by appealing to the defining properties of the $\mathbf{G}$ and $\mathbf{C}$ dyadics, the surface integral in (C3) can be evaluated in closed form, with the result (depicted schematically in Fig. 6)

$$
\begin{aligned}
& \overline{\mathcal{I}}_{i k l}\left(\mathbf{r}, \mathbf{r}^{\prime}\right) \\
& = \begin{cases}0, & \text { if both } \mathbf{r}, \mathbf{r}^{\prime} \text { lie inside } \mathcal{C}, \\
\frac{\partial}{\partial \mathbf{r}_{i}} G_{k l}\left(\mathbf{r}-\mathbf{r}^{\prime}\right), & \text { if } \mathbf{r} \text { lies inside and } \mathbf{r}^{\prime} \text { lies outside } \mathcal{C}, \\
-\frac{\partial}{\partial \mathbf{r}_{i}} G_{k l}\left(\mathbf{r}-\mathbf{r}^{\prime}\right), & \text { if } \mathbf{r} \text { lies outside and } \mathbf{r}^{\prime} \text { lies inside } \mathcal{C}, \\
0, & \text { if both } \mathbf{r}, \mathbf{r}^{\prime} \text { lie outside } \mathcal{C} .\end{cases}
\end{aligned}
$$

[As far as we can tell, the symmetrization in Eq. (C3) is necessary to achieve the compact form of Eq. (C5); our attempts to evaluate the nonsymmetrized $\mathcal{I}$ in concise form were unsuccessful.]

For the purposes of this appendix it is convenient to work in length units such that $\kappa=1$. With this convention, the $\mathbf{G}$ and $\mathbf{C}$ dyadics are related to the scalar Green's function for the Helmholtz equation according to

$$
G_{i j}=\left[\delta_{i j}-\partial_{i} \partial_{j}\right] G_{0}, \quad C_{i j}=-\varepsilon_{i j k} \partial_{k} G_{0},
$$




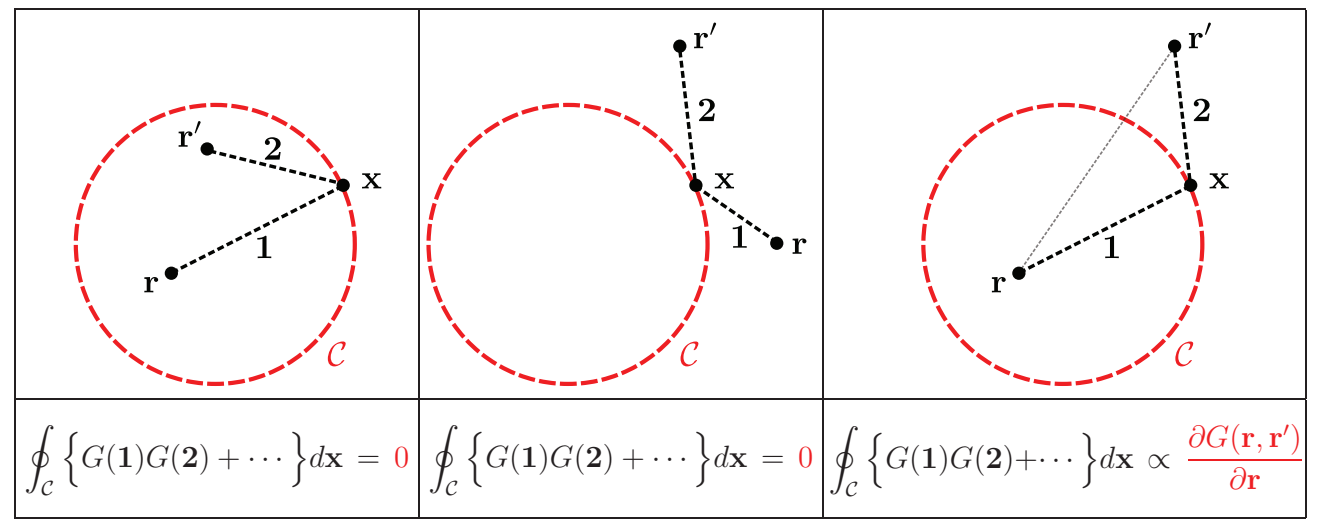

FIG. 6. (Color online) Schematic summary of the integral identity (C5) proved in Appendix C. We consider a closed bounding surface $\mathcal{C}$ and choose two points $\mathbf{r}$ and $\mathbf{r}^{\prime}$, which may lie both inside (left panel), both outside (center panel), or on opposite sides (right panel) of $\mathcal{C}$. We write an expression [Eq. (C4)] that involves products of DGFs, one connecting $\mathbf{r}$ to a point $\mathbf{x}$ on $\mathcal{C}$ and a second connecting $\mathbf{x}$ to $\mathbf{r}^{\prime}$. Then we evaluate the surface integral of this expression as $\mathbf{x}$ ranges over all of $\mathcal{C}$. The answer we obtain depends on the relative positioning of $\mathbf{r}$ and $\mathbf{r}^{\prime}$ with respect to $\mathcal{C}$ : If the two points lie both inside or both outside $\mathcal{C}$, the surface integral vanishes, while if the two points lie on opposite sides of $\mathcal{C}$ then the surface integral yields the derivative of a DGF connecting $\mathbf{r}$ to $\mathbf{r}^{\prime}$.

and $G_{0}$ satisfies

$$
\left[\partial_{A} \partial_{A}-1\right] G_{0}(\mathbf{r})=\delta(\mathbf{r}) .
$$

The evaluation of the surface integral (C3) now proceeds in several stages.

\section{Apply divergence theorem}

The first step is to recast the surface integral in (C3) as a volume integral over the volume bounded by $\mathcal{C}$,

$$
\begin{aligned}
\oint_{\mathcal{C}} \bar{V}_{A} n_{A} d A & =\int_{\mathcal{V}} \partial_{A} \bar{V}_{A} d V \quad(\mathcal{C}=\partial \mathcal{V}) \\
& =\int_{\mathcal{V}} W d V,
\end{aligned}
$$

where we put $W \equiv \partial_{A} \bar{V}_{A}$.

Each of the six terms in $\bar{V}_{A}$ contains two factors and hence contributes two terms to $W$ (by the chain rule for differentiation). Terms of the form $\partial_{A} C_{i A}$ vanish; to each of the remaining (nonvanishing) terms we assign a label, as tabulated in Table I. Note that derivatives of $G_{i j}^{\mathbf{2}}$ enter with a minus sign,

TABLE I. Terms in $W \equiv \partial_{A} \bar{V}_{A}$.

\begin{tabular}{lc}
\hline \hline Label & Term \\
\hline$W^{\mathbf{1}}$ & {$\left[\partial_{A} G_{i k}^{\mathbf{1}}\right]\left[G_{l A}^{2}\right]$} \\
$W^{\mathbf{2}}$ & $-\left[G_{i k}^{1}\right]\left[\partial_{A} G_{l A}^{2}\right]$ \\
$W^{\mathbf{3}}$ & {$\left[\partial_{A} G_{k A}^{1}\right]\left[G_{i l}^{2}\right]$} \\
$W^{4}$ & $-\left[G_{k A}^{1}\right]\left[\partial_{A} G_{i l}^{2}\right]$ \\
$W^{\mathbf{5}}$ & $-\left[\partial_{i} G_{k A}^{1}\right]\left[G_{l A}^{2}\right]$ \\
$W^{\mathbf{6}}$ & {$\left[G_{k A}^{1}\right]\left[\partial_{i} G_{l A}^{2}\right]$} \\
$W^{7}$ & $-\left[\partial_{A} C_{i k}^{1}\right]\left[C_{l A}^{1}\right]$ \\
$W^{\mathbf{8}}$ & {$\left[C_{k A}^{1}\right]\left[\partial_{A} C_{i l}^{2}\right]$} \\
$W^{\mathbf{9}}$ & {$\left[\partial_{i} C_{A k}^{1}\right]\left[C_{l A}^{2}\right]$} \\
$W^{\mathbf{1 0}}$ & $-\left[C_{A k}^{1}\right]\left[\partial_{i} C_{l A}^{2}\right]$ \\
\hline \hline
\end{tabular}

because we are differentiating with respect to $\mathbf{x}$, which enters the argument of $G_{i j}^{2}$ with a minus sign.

\section{Treatment of $W^{2}$ and $W^{3}$}

We first consider the terms labeled $W^{2}$ and $W^{3}$ in Table I. Starting with the first of these, we have

$$
W^{2}=-\left[G_{i k}^{\mathbf{1}}\right]\left[\partial_{A} G_{l A}^{2}\right] .
$$

Expand the second factor using (C6):

$$
=-\left[G_{i k}^{\mathbf{1}}\right]\left[\partial_{l}\left(1-\partial_{A} \partial_{A}\right) G_{0}^{2}\right] .
$$

Apply (C7):

$$
=+\left[G_{i k}^{\mathbf{1}}\right]\left[\partial_{l} \delta(\mathbf{2})\right] .
$$

Integrate by parts:

$$
\sim+\left[\partial_{l} G_{i k}^{\mathbf{1}}\right][\delta(\mathbf{2})],
$$

where $\sim$ means "equivalent as long as we are underneath the volume-integration sign' and $\delta(\mathbf{2})$ is shorthand for $\delta\left(\mathbf{r}^{\prime}-\mathbf{x}\right)$. (Note that the minus sign coming from the integration by parts is canceled by the minus sign coming from the fact that $\mathbf{x}$ enters 2 with a minus sign, as noted above.)

By analogous operations, we find

$$
W^{\mathbf{3}} \sim-[\delta(\mathbf{1})]\left[\partial_{k} G_{i l}^{2}\right],
$$

where $\delta(\mathbf{1})$ is shorthand for $\delta(\mathbf{x}-\mathbf{r})$. We now set aside results (C8) and (C9) for future use.

\section{Treatment of remaining terms}

\section{a. Rewrite in terms of $G_{0}$}

Turning next to the remaining eight terms in Table I, we begin by using (C6) to rewrite everything in terms of the scalar 
TABLE II. Terms in $X$.

\begin{tabular}{lc}
\hline \hline Label & Term \\
\hline$X^{\mathbf{1}}$ & $-\delta_{k l}\left[\partial_{i} G_{0}^{\mathbf{1}}\right]\left[G_{0}^{\mathbf{2}}\right]$ \\
$X^{\mathbf{2}}$ & {$\left[\partial_{i} G_{0}^{\mathbf{1}}\right]\left[\partial_{k} \partial_{l} G_{0}^{\mathbf{2}}\right]$} \\
$X^{\mathbf{3}}$ & $\delta_{i k}\left[\partial_{l} G_{0}^{\mathbf{1}}\right]\left[G_{0}^{\mathbf{2}}\right]$ \\
$X^{\mathbf{4}}$ & $-\delta_{i k}\left[\partial_{A} G_{0}^{\mathbf{1}}\right]\left[\partial_{A} \partial_{l} G_{0}^{\mathbf{2}}\right]$ \\
$X^{\mathbf{5}}$ & $-\delta_{i l}\left[G_{0}^{\mathbf{1}}\right]\left[\partial_{k} G_{0}^{\mathbf{2}}\right]$ \\
$X^{\mathbf{6}}$ & $\delta_{k l}\left[G_{0}^{\mathbf{1}}\right]\left[\partial_{i} G_{0}^{\mathbf{2}}\right]$ \\
$X^{\mathbf{7}}$ & $\delta_{i l}\left[\partial_{A} \partial_{k} G_{0}^{\mathbf{1}}\right]\left[\partial_{A} G_{0}^{\mathbf{2}}\right]$ \\
$X^{\mathbf{8}}$ & $-\left[\partial_{k} \partial_{l} G_{0}^{\mathbf{1}}\right]\left[\partial_{i} G_{0}^{\mathbf{2}}\right]$ \\
$X^{\mathbf{9}}$ & $-\delta_{k l}\left[\partial_{A} \partial_{A} G_{0}^{\mathbf{1}}\right]\left[\partial_{i} G_{0}^{\mathbf{2}}\right]$ \\
$X^{\mathbf{1 0}}$ & $+\partial_{i l}\left[\partial_{A} \partial_{A} G_{0}^{\mathbf{1}}\right]\left[\partial_{k} G_{0}^{\mathbf{2}}\right]$ \\
$X^{\mathbf{1 1}}$ & $+\delta_{k l}\left[\partial_{i} G_{0}^{\mathbf{1}}\right]\left[\partial_{A} \partial_{A} G_{0}^{\mathbf{2}}\right]$ \\
$X^{\mathbf{1 2}}$ & $-\delta_{i k}\left[\partial_{l} G_{0}^{\mathbf{1}}\right]\left[\partial_{A} \partial_{A} G_{0}^{\mathbf{2}}\right]$ \\
$X^{\mathbf{1 3}}$ & $-\delta_{i l}\left[\partial_{A} \partial_{k} G_{0}^{\mathbf{1}}\right]\left[\partial_{A} G_{0}^{\mathbf{2}}\right]$ \\
$X^{\mathbf{1 4}}$ & $+\delta_{i k}\left[\partial_{A} G_{0}^{\mathbf{1}}\right]\left[\partial_{l} \partial_{A} G_{0}^{\mathbf{2}}\right]$ \\
$X^{\mathbf{1 5}}$ & $-\left[\partial_{i} G_{0}^{\mathbf{1}}\right]\left[\partial_{k} \partial_{l} G_{0}^{\mathbf{2}}\right]$ \\
$X^{\mathbf{1 6}}$ & {$\left[\partial_{k} \partial_{l} G_{0}^{\mathbf{1}}\right]\left[\partial_{i} G_{0}^{\mathbf{2}}\right]$} \\
\hline \hline
\end{tabular}

Green's function:

$$
\begin{aligned}
W^{\mathbf{1}} & +W^{\mathbf{4}}+W^{\mathbf{5}}+W^{\mathbf{6}} \\
= & {\left[\partial_{A} G_{i k}^{\mathbf{1}}-\partial_{i} G_{k A}^{\mathbf{1}}\right]\left[G_{l A}^{\mathbf{2}}\right]+\left[G_{k A}^{\mathbf{1}}\right]\left[\partial_{i} G_{l A}^{\mathbf{2}}-\partial_{A} G_{i l}^{\mathbf{2}}\right] } \\
= & {\left[\delta_{i k} \partial_{A} G_{0}^{\mathbf{1}}-\delta_{k A} \partial_{i} G_{0}^{\mathbf{1}}\right]\left[\delta_{l A} G_{0}^{\mathbf{2}}-\partial_{l} \partial_{A} G_{0}^{\mathbf{2}}\right] } \\
& +\left[\delta_{k A} G_{0}^{\mathbf{1}}-\partial_{k} \partial_{A} G_{0}^{\mathbf{1}}\right]\left[\delta_{l A} \partial_{i} G_{0}^{\mathbf{2}}-\delta_{i l} \partial_{A} G_{0}^{\mathbf{2}}\right], \\
W^{\mathbf{7}}+ & W^{\mathbf{8}}+W^{\mathbf{9}}+W^{\mathbf{1 0}} \\
= & -\varepsilon_{i k B} \varepsilon_{l A C}\left[\partial_{A} \partial_{B} G_{0}^{\mathbf{1}}\right]\left[\partial_{C} G_{0}^{\mathbf{2}}\right]+\varepsilon_{k A B} \varepsilon_{i l C}\left[\partial_{B} G_{0}^{\mathbf{1}}\right]\left[\partial_{A} \partial_{C} G_{0}^{\mathbf{2}}\right] \\
& +\varepsilon_{A k B} \varepsilon_{l A C}\left[\partial_{i} \partial_{B} G_{0}^{\mathbf{1}}\right]\left[\partial_{C} G_{0}^{\mathbf{2}}\right]-\varepsilon_{A k B} \varepsilon_{l A C}\left[\partial_{B} G_{0}^{\mathbf{1}}\right]\left[\partial_{i} \partial_{C} G_{0}^{\mathbf{2}}\right] .
\end{aligned}
$$

We can trade Levi-Civita symbols for Kronecker $\delta$ 's using the standard identity

$$
\begin{aligned}
\varepsilon_{A B C} \varepsilon_{D E F}= & \delta_{A D}\left[\delta_{B E} \delta_{C F}-\delta_{B F} \delta_{C E}\right] \\
& +\delta_{A E}\left[\delta_{B F} \delta_{C D}-\delta_{B D} \delta_{C F}\right] \\
& +\delta_{A F}\left[\delta_{B D} \delta_{C E}-\delta_{B E} \delta_{C D}\right] .
\end{aligned}
$$

We then find

$$
\begin{aligned}
W^{\mathbf{7}}+ & W^{\mathbf{8}}+W^{\mathbf{9}}+W^{\mathbf{1 0}} \\
= & {\left[\partial_{A} \partial_{A} G_{0}^{\mathbf{1}}\right]\left[\delta_{i l} \partial_{k} G_{0}^{\mathbf{2}}-\delta_{k l} \partial_{i} G_{0}^{\mathbf{2}}\right] } \\
& -\left[\delta_{i k} \partial_{l} G_{0}^{\mathbf{1}}-\delta_{k l} \partial_{i} G_{0}^{\mathbf{1}}\right]\left[\partial_{A} \partial_{A} G_{0}^{\mathbf{2}}\right] \\
& -\delta_{i l}\left[\partial_{k} \partial_{A} G_{0}^{\mathbf{1}}\right]\left[\partial_{A} G_{0}^{\mathbf{2}}\right]+\delta_{i k}\left[\partial_{A} G_{0}^{\mathbf{1}}\right]\left[\partial_{l} \partial_{A} G_{0}^{\mathbf{2}}\right] \\
& -\left[\partial_{i} G_{0}^{\mathbf{1}}\right]\left[\partial_{k} \partial_{l} G_{0}^{\mathbf{2}}\right]+\left[\partial_{k} \partial_{l} G_{0}^{\mathbf{1}}\right]\left[\partial_{i} G_{0}^{\mathbf{2}}\right] .
\end{aligned}
$$

\section{b. Label individual terms}

To proceed, we now assign a label to each separate term in (C10) and (C11):

$W^{1}+W^{4}+W^{5}+W^{6}+W^{7}+W^{8}+W^{9}+W^{10}=\sum_{n=1}^{16} X^{\mathbf{n}}$.

The precise forms of the $X^{n}$ terms are detailed in Table II.

\section{c. Recombine terms}

We first note the obvious cancellations:

$$
\begin{array}{ll}
X^{2}+X^{15}=0, & X^{4}+X^{14}=0, \\
X^{7}+X^{13}=0, & X^{8}+X^{16}=0 .
\end{array}
$$

Summing and appropriately recombining the remaining terms, we find

$$
\begin{aligned}
& X^{\mathbf{1}}+X^{\mathbf{3}}+X^{\mathbf{1 1}}+X^{\mathbf{1 2}} \\
& \quad=\left[\delta_{k l} \partial_{i} G_{0}^{\mathbf{1}}-\delta_{i k} \partial_{l} G_{0}^{\mathbf{1}}\right]\left[\partial_{A} \partial_{A} G_{0}^{\mathbf{2}}-G_{0}^{\mathbf{2}}\right]
\end{aligned}
$$

and

$$
\begin{aligned}
& X^{\mathbf{5}}+X^{\mathbf{6}}+X^{\mathbf{9}}+X^{\mathbf{1 0}} \\
& \quad=\left[\partial_{A} \partial_{A} G_{0}^{\mathbf{1}}-G_{0}^{\mathbf{1}}\right]\left[\delta_{i l} \partial_{k} G_{0}^{\mathbf{1}}-\delta_{k l} \partial_{i} G_{0}^{\mathbf{1}}\right] .
\end{aligned}
$$

From (C6) it follows that

$$
\left[\delta_{k l} \partial_{i} G_{0}-\delta_{i k} \partial_{l} G_{0}\right]=\left[\partial_{i} G_{k l}-\partial_{l} G_{i k}\right] .
$$

Using this and (C7), we can rewrite the previous two equations in the form

$$
X^{1}+X^{3}+X^{11}+X^{12}=\left[\partial_{i} G_{k l}^{1}-\partial_{l} G_{i k}^{\mathbf{1}}\right][\delta(2)]
$$

and

$$
X^{5}+X^{6}+X^{9}+X^{10}=[\delta(1)]\left[\partial_{k} G_{i l}^{2}-\partial_{i} G_{k l}^{2}\right] .
$$

\section{Final steps}

Finally, we combine Eqs. (C8), (C9), (C12), and (C13) to obtain

$$
\begin{aligned}
\overline{\mathcal{I}}_{i k l}= & \int_{\mathcal{V}}\left\{W^{2}+W^{3}+X^{\mathbf{1}}+X^{\mathbf{3}}+X^{\mathbf{1 1}}+X^{\mathbf{1 2}}\right. \\
& \left.+X^{\mathbf{5}}+X^{\mathbf{6}}+X^{\mathbf{9}}+X^{\mathbf{1 0}}\right\} d \mathbf{x} \\
= & \int_{\mathcal{V}}\left\{\left[\partial_{i} G_{k l}^{\mathbf{1}}\right] \delta(\mathbf{2})-\delta(\mathbf{1})\left[\partial_{i} G_{k l}^{\mathbf{2}}\right]\right\} d \mathbf{x} .
\end{aligned}
$$

Writing out the function arguments, this reads

$$
\begin{aligned}
\overline{\mathcal{I}}_{i k l}\left(\mathbf{r}, \mathbf{r}^{\prime}\right)= & \int_{\mathcal{V}}\left\{\left[\partial_{i} G_{k l}(\mathbf{x}-\mathbf{r})\right] \delta\left(\mathbf{r}^{\prime}-\mathbf{x}\right)\right. \\
& \left.-\delta(\mathbf{x}-\mathbf{r})\left[\partial_{i} G_{k l}\left(\mathbf{r}^{\prime}-\mathbf{x}\right)\right]\right\} d \mathbf{x} .
\end{aligned}
$$

We now proceed on a case-by-case basis depending on the positions of $\mathbf{r}, \mathbf{r}^{\prime}$.

(i) First, if $\mathbf{r}$ and $\mathbf{r}^{\prime}$ both lie outside the bounding surface $\mathcal{C}$, then neither $\delta$ function contributes and we have $\overline{\mathcal{I}}=0$.

(ii) If $\mathbf{r}$ lies inside $\mathcal{C}$ while $\mathbf{r}^{\prime}$ lies outside $\mathcal{C}$, then only the second $\delta$ function contributes, and we find

$$
\mathcal{I}_{i k l}\left(\mathbf{r}, \mathbf{r}^{\prime}\right)=-\partial_{i} G_{k l}\left(\mathbf{r}^{\prime}-\mathbf{r}\right)=+\partial_{i} G_{k l}\left(\mathbf{r}-\mathbf{r}^{\prime}\right) .
$$

(iii) If $\mathbf{r}$ lies outside $\mathcal{C}$ while $\mathbf{r}^{\prime}$ lies inside $\mathcal{C}$, then only the first $\delta$ function contributes, and we find

$$
\mathcal{I}_{i k l}\left(\mathbf{r}, \mathbf{r}^{\prime}\right)=\partial_{i} G_{k l}\left(\mathbf{r}^{\prime}-\mathbf{r}\right)=-\partial_{i} G_{k l}\left(\mathbf{r}-\mathbf{r}^{\prime}\right) .
$$

(iv) Finally, if both $\mathbf{r}$ and $\mathbf{r}^{\prime}$ lie inside $\mathcal{C}$ then both $\delta$ functions contribute, their contributions cancel, and we find $\overline{\mathcal{I}}=0$.

The result (C5) is thus established, and our proof is complete. 


\section{The $\overline{\mathcal{J}}$ kernel}

The development of Sec. IIC also makes reference to a version of the $\mathcal{I}$ kernel defined in analogy to Eqs. (C1) and (C2), but with the " $G G-C C$ " structure of (C2) replaced with a " $C G+G C$ " structure:

$$
\begin{aligned}
\mathcal{J}_{i k l}\left(\mathbf{r}, \mathbf{r}^{\prime}\right)= & \kappa^{2} \oint_{\mathcal{C}} Y_{A}\left(\mathbf{r}, \mathbf{r}^{\prime}, \mathbf{x}\right) n_{A}(\mathbf{x}) d \mathbf{x}, \\
Y_{A}\left(\mathbf{r}, \mathbf{r}^{\prime}, \mathbf{x}\right)= & C_{i k}^{\mathbf{1}} G_{l A}^{\mathbf{2}}-\frac{\delta_{i A}}{2} C_{B k}^{\mathbf{1}} G_{l B}^{\mathbf{2}}+G_{i k}^{\mathbf{1}} C_{l A}^{\mathbf{2}} \\
& -\frac{\delta_{i A}}{2} G_{B k}^{\mathbf{1}} C_{l B}^{\mathbf{2}} .
\end{aligned}
$$

We also define a symmetrized version defined in analogy to (C3):

$$
\overline{\mathcal{J}}_{i k l}\left(\mathbf{r}, \mathbf{r}^{\prime}\right)=\mathcal{J}_{i k l}\left(\mathbf{r}, \mathbf{r}^{\prime}\right)+\mathcal{J}_{i l k}\left(\mathbf{r}^{\prime}, \mathbf{r}\right) .
$$

In Appendix A we noted that the curl operation takes $\mathbf{G}$ into $\mathbf{C}$ and $\mathbf{C}$ into $\mathbf{- G}$. [Technically, in the latter case there is an additional $\delta$ function, which we neglect for reasons discussed in conjunction with Eqs. (40) above.] With this observation we see that $(\mathrm{C} 16)$ is obtained from (C2) simply by taking the curl with respect to the $k$ index and thus that the $\overline{\mathcal{J}}$ kernel is the result of the same operation applied to the $\overline{\mathcal{I}}$ kernel:

$$
\begin{aligned}
& \overline{\mathcal{J}}_{i k l}\left(\mathbf{r}, \mathbf{r}^{\prime}\right) \\
& =\frac{1}{\kappa} \varepsilon_{k A B} \partial_{A} \overline{\mathcal{I}}_{i B l}\left(\mathbf{r}, \mathbf{r}^{\prime}\right) \\
& = \begin{cases}0, & \text { if both } \mathbf{r}, \mathbf{r}^{\prime} \text { lie inside } \mathcal{C}, \\
\frac{\partial}{\partial \mathbf{r}_{i}} C_{k l}\left(\mathbf{r}-\mathbf{r}^{\prime}\right), & \text { if } \mathbf{r} \text { lies inside and } \mathbf{r}^{\prime} \text { lies outside } \mathcal{C}, \\
-\frac{\partial}{\partial \mathbf{r}_{i}} C_{k l}\left(\mathbf{r}-\mathbf{r}^{\prime}\right), & \text { if } \mathbf{r} \text { lies outside and } \mathbf{r}^{\prime} \text { lies inside } \mathcal{C}, \\
0, & \text { if both } \mathbf{r}, \mathbf{r}^{\prime} \text { lie outside } \mathcal{C} .\end{cases}
\end{aligned}
$$

\section{APPENDIX D: A REVIEW OF CONSTRAINED PATH-INTEGRAL TECHNIQUES FOR CASIMIR ENERGIES}

The path-integral derivation of the FSC Casimir formulas presented in Sec. III follows earlier work on path-integral formulations of field-fluctuation problems. These techniques were pioneered by Bordag, Robaschik, and Wieczorek [39] and by $\mathrm{Li}$ and Kardar $[66,67]$ and have since been further developed by a number of authors (see $[2,68]$ for extensive surveys of recent developments.) This appendix presents a brief review of the key steps in this approach.

\section{Casimir energies from constrained path integrals}

In the presence of material boundaries, the partition function for a quantum field $\phi$ (which may be scalar, vector, electromagnetic, or otherwise, but is here assumed bosonic) at inverse temperature $\beta$ takes the form

$$
Z(\beta)=\int[\mathcal{D} \phi(\tau, \mathbf{x})]_{C} e^{-\frac{1}{\hbar} S_{\beta}[\phi]},
$$

where the action $S_{\beta}$ is the spacetime integral of the Euclidean Lagrangian density for the $\phi$ field,

$$
S_{\beta}[\phi]=\int_{0}^{\hbar \beta} d \tau \int d \mathbf{x} \mathcal{L}_{E}\{\phi(\tau, \mathbf{x})\},
$$

and where the notation $[\cdots]_{C}$ in (D1) indicates that this is a constrained path integral, in which the functional integration extends only over field configurations $\phi$ satisfying the appropriate boundary conditions at all material boundaries.

If the boundary conditions are time independent and the Lagrangian density contains no terms of higher-than-quadratic order in $\phi$ and its derivatives, then it is convenient to introduce a Fourier series in the Euclidean time variable,

$$
\phi(\tau, \mathbf{x})=\sum_{n} \phi_{n}(\mathbf{x}) e^{-i \xi_{n} \tau}, \quad \xi_{n}=\frac{2 \pi n}{\hbar \beta},
$$

whereupon the path integral (D1) factorizes into a product of contributions from individual frequencies,

$$
\begin{gathered}
Z(\beta)=\prod_{n} \mathcal{Z}\left(\beta ; \xi_{n}\right), \\
\mathcal{Z}\left(\beta ; \xi_{n}\right)=\int\left[\mathcal{D} \phi_{n}(\mathbf{x})\right]_{C} e^{-S\left[\phi_{n} ; \xi_{n}\right]},
\end{gathered}
$$

with

$$
S\left[\phi_{n}(\mathbf{x}) ; \xi_{n}\right]=\beta \int d \mathbf{x} \mathcal{L}_{E}\left\{\phi_{n}(\mathbf{x}) e^{-i \xi_{n} \tau}\right\}
$$

representing the contribution to the full action (D2) made only by those field configurations with Euclidean-time dependence $\sim e^{-i \xi_{n} \tau}$. The free energy is then obtained as a sum over Matsubara frequencies,

$$
F=-\frac{1}{\beta} \ln \frac{Z(\beta)}{Z_{\infty}(\beta)}=-\frac{1}{\beta} \sum_{n=0}^{\infty} \ln \frac{\mathcal{Z}\left(\beta, \xi_{n}\right)}{\mathcal{Z}_{\infty}\left(\beta, \xi_{n}\right)},
$$

where $Z_{\infty}\left(\mathcal{Z}_{\infty}\right)$ is $Z(\mathcal{Z})$ evaluated with all material objects separated by infinite distances [dividing out these contributions in (D4) is a useful convention that amounts to a choice of the zero of energy]. In the zero-temperature limit, the frequency sum becomes an integral, and the zero-temperature Casimir energy is

$$
\mathcal{E}=-\frac{\hbar}{2 \pi} \int_{0}^{\infty} d \xi \ln \frac{\mathcal{Z}(\xi)}{\mathcal{Z}_{\infty}(\xi)} .
$$

(Here and below we omit the $\beta$ argument to $\mathcal{Z}$.)

\section{Enforcing constraints via functional $\delta$ functions}

Equations (D4) and (D5) reduce the computation of Casimir energies to the evaluation of constrained path integrals (D3). In most branches of physics, the path integrals associated with physically interesting quantities are difficult to evaluate because the action $S$ in the exponent contains interaction terms (terms of third or higher order in the fields and their derivatives). In Casimir physics, on the other hand, the action is not more than quadratic in $\phi$, and the difficulty in evaluating expressions like (D3) stems instead from the challenge of implementing the implicit constraint on the functional integration measure, arising from the boundary conditions and indicated by the $[\cdots]_{C}$ notation in (D3).

The innovation of Bordag [39] and of Li and Kardar [66] was to represent these constraints explicitly through the use of functional $\delta$ functions. If the boundary conditions on $\phi$ may be expressed as the vanishing of a set of quantities $\left\{L_{\alpha} \phi\right\}$, 
where $\left\{L_{\alpha}\right\}$ will generally be some family of linear integrodifferential operators indexed by a discrete or continuous label $\alpha$, then the constrained path integral may be written in the form

$$
\begin{aligned}
\mathcal{Z}\left(\xi_{n}\right) & =\int\left[\mathcal{D} \phi_{n}(\mathbf{x})\right]_{C} e^{-S\left[\phi_{n} ; \xi_{n}\right]} \\
& =\int \mathcal{D} \phi_{n}(\mathbf{x}) \prod_{\alpha} \delta\left(L_{\alpha} \phi\right) e^{-S\left[\phi_{n} ; \xi_{n}\right]},
\end{aligned}
$$

where now the functional integration over $\phi_{n}$ is unconstrained. A particularly convenient representation for the one-dimensional Dirac $\delta$ function is

$$
\delta(u)=\int \frac{d \lambda}{2 \pi} e^{i \lambda u},
$$

where we may think of $\lambda$ as a Lagrange multiplier enforcing the constraint that $u$ vanish. Inserting one copy of (D7) for each $\delta$ function in the product in (D6) yields

$$
\mathcal{Z}\left(\xi_{n}\right)=\int \mathcal{D} \phi_{n}(\mathbf{x}) \int \prod_{\alpha} \frac{d \lambda_{\alpha}}{2 \pi} e^{-S\left[\phi_{n} ; \xi_{n}\right]+i \sum_{\alpha} \lambda_{\alpha} L_{\alpha} \phi} .
$$

The final step is to evaluate the unconstrained integral over $\phi$; since the exponent is quadratic in $\phi$, this can be done exactly using standard techniques of Gaussian integration, yielding an expression of the form

$$
\mathcal{Z}\left(\xi_{n}\right)=\{\#\} \int \prod_{\alpha} d \lambda_{\alpha} e^{-S^{\mathrm{eff}}\left\{\lambda_{\alpha}\right\}}
$$

[where $\{\#\}$ is a constant that cancels in the ratios in (D4) and (D5)]. The constrained functional integral over the field $\phi$ is thus replaced with a new integral over the set of Lagrange multipliers $\left\{\lambda_{\alpha}\right\}$, with an effective action $S^{\text {eff }}$ describing interactions mediated by the original fluctuating field $\phi$.

\section{Representation of boundary conditions}

Equation (D8) makes clear that the practical convenience of path-integral Casimir computations is entirely determined by the choice of the Lagrange multipliers $\left\{\lambda_{\alpha}\right\}$ and the complexity of their effective action $S^{\text {eff }}$; these, in turn, depend on the details of the boundary conditions imposed on the fluctuating field. For a given physical situation there may be multiple ways to express the boundary conditions, each of which will generally lead to a distinct expression for the integral in (D8). Ultimately, of course, all choices must lead to equivalent results, but different choices may exhibit significant differences in computational complexity and in the range of geometries that can be efficiently treated. Several different representations of boundary conditions and Lagrange multipliers have appeared in the literature to date.

The original work of Bordag et al. [39] considered QED in the presence of superconducting boundaries, with the boundary conditions taken to be the vanishing of the normal components of the dual field-strength tensor; in the notation of the previous section, $L_{\mathbf{x}} \phi=\hat{n}^{\mu} F_{\mu \nu}^{*}(\mathbf{x})$, and the set of Lagrange multipliers $\left\{\lambda_{\mathbf{x}}\right\}$ constitutes a three-component auxiliary field defined on the bounding surfaces. The method is applicable to the computation of electromagnetic Casimir energies, but the treatment was restricted to the case of parallel planar boundaries.
Li and Kardar [66,67] considered a scalar field satisfying Dirichlet or Neumann boundary conditions on a prescribed boundary manifold. Here again the boundary conditions amount to the vanishing of a local operator applied to $\phi$, $L_{\mathbf{x}} \phi=\phi(\mathbf{x})$ (Dirichlet) or $L_{\mathbf{x}} \phi=|\partial \phi / \partial n|_{\mathbf{x}}$ (Neumann), and we have one Lagrange multiplier $\lambda(\mathbf{x})$ for each point on the boundary manifold. In this case it is tempting to interpret $\lambda(\mathbf{x})$ as a scalar source density, confined to the boundary surfaces and with a self-interaction induced by the fluctuations of the $\phi$ field. This formulation was capable, in principle, of handling arbitrarily shaped boundary surfaces, but was restricted to the case of scalar fields.

The technique of Refs. [66,67] was subsequently reformulated $[2,44,69]$ in a way that allowed extension to the case of the electromagnetic field. Whereas the original formulation imposed a local form of the boundary conditions - and took the Lagrange multipliers $\lambda(\mathbf{x})$ to be local surface quantities- the revised formulation abandons the surface-source picture in favor of an alternative viewpoint emphasizing incoming and outgoing electromagnetic waves. This approach associates one Lagrange multiplier $\lambda_{\alpha}$ to each multipole term in a multipole expansion of the EM field, with the choice of multipole basis (spherical, cylindrical, etc.) governed by the symmetries of the problem; the effective action $S^{\text {eff }}$ then describes the interactions among multipoles.

The virtue of multipole expansions is that, for certain geometries, a small number of multipole coefficients may suffice to solve many problems of interest to high accuracy. This has long been understood in domains such as electrostatics and scattering theory, and in recent years has been impressively demonstrated in the Casimir context as well [2,44,69], where multipole expansions have been used to obtain rapidly convergent and even analytically tractable series for Casimir energies in certain special geometries. The trick, of course, is that the very definition of the multipoles already encodes a significant amount of information about the geometry, thus requiring relatively little additional work to pin down what more remains to be said in any particular situation.

However, this blessing becomes a curse when we seek a unified formalism capable of treating all geometries on an equal footing. The very geometric specificity of the multipole description, which so streamlines the treatment of compatible or nearly compatible geometries, has the opposite effect of complicating the treatment of incompatible geometries; thus, whereas a basis of spherical multipoles might allow highly efficient treatment of interacting spheres or nearly spherical bodies, it would be a particularly unwieldy choice for the description of cylinders, tetrahedra, or parallelepipeds. Of course, for each new geometric configuration we could simply redefine our multipole expansion and correspondingly reimplement the full arsenal of computational machinery (a strategy pursued for a dizzying array of geometries in Ref. [2]), but such a procedure contradicts the spirit of a single, general-purpose scheme into which we simply plug an arbitrary experimental geometry and turn a crank.

Instead, the goal of designing a more general-purpose implementation of the path-integral Casimir paradigm leads us to seek a representation of the boundary conditions that, while inevitably less efficient than spherical multipoles for spheres (or cylindrical multipoles for cylinders, or ...), has the 
flexibility to handle all manner of surfaces within a single computational framework. This is one motivation for the
FSC approach to Casimir computations, whose path-integral derivation we presented in Sec. III.
[1] S. G. Johnson, in Casimir Physics, edited by D. Dalvit, P. Milonni, D. Roberts, and F. da Rosa, Lecture Notes in Physics Vol. 834, (Springer, Berlin, 2011), Chap. 6, pp. 175-218.

[2] S. J. Rahi, T. Emig, N. Graham, R. L. Jaffe, and M. Kardar, Phys. Rev. D 80, 085021 (2009).

[3] http://homerreid.com/scuff-EM.

[4] M. T. Homer Reid, A. W. Rodriguez, J. White, and S. G. Johnson, Phys. Rev. Lett. 103, 040401 (2009).

[5] M. T. Homer Reid, J. White, and S. G. Johnson, Phys. Rev. A 84, 010503(R) (2011).

[6] M. Levin, A. P. McCauley, A. W. Rodriguez, M. T. Homer Reid, and S. G. Johnson, Phys. Rev. Lett. 105, 090403 (2010).

[7] K. Pan, A. P. McCauley, A. W. Rodriguez, M. T. Homer Reid, J. K. White, and S. G. Johnson, Phys. Rev. A 83, 040503(R) (2011).

[8] A. P. McCauley, A. W. Rodriguez, M. T. Homer Reid, and S. G. Johnson, arXiv:1105.0404.

[9] L. P. Pitaevskii, Phys. Rev. A 73, 047801 (2006).

[10] T. G. Philbin, New J. Phys. 12, 123008 (2010).

[11] T. G. Philbin, New J. Phys. 13, 063026 (2011).

[12] K. A. Milton and J. Wagner, Phys. Rev. D 77, 045005 (2008).

[13] J. D. Jackson, Classical Electrodynamics, 3rd ed. (Wiley, New York, 1998).

[14] G. Bimonte, Phys. Rev. A 80, 042102 (2009).

[15] H. B. G. Casimir, Proc. K. Ned. Akad. Wet. 51, 793 (1948).

[16] S. K. Lamoreaux, Phys. Rev. Lett. 78, 5 (1997).

[17] F. Capasso, J. N. Munday, D. Iannuzzi, and H. B. Chan, IEEE. J. Sel. Top. Quantum Electron. 13, 400 (2007).

[18] J. N. Munday, F. Capasso, and V. Parsegian, Nature (London) 457, 170 (2009).

[19] H.-C. Chiu, G. L. Klimchitskaya, V. N. Marachevsky, V. M. Mostepanenko, and U. Mohideen, Phys. Rev. B 81, 115417 (2010).

[20] A. W. Rodriguez, F. Capasso, and S. G. Johnson, Nat. Photon. 5, $211(2011)$

[21] C. Genet, A. Lambrecht, and S. Reynaud, Phys. Rev. A 67, 043811 (2003).

[22] Paulo A. Maia Neto, A. Lambrecht, and S. Reynaud, Phys. Rev. A 72, 012115 (2005).

[23] A. Lambrecht, P. A. M. Neto, and S. Reynaud, New J. Phys. 8, 243 (2006).

[24] O. Kenneth and I. Klich, Phys. Rev. B 78, 014103 (2008).

[25] P. S. Davids, F. Intravaia, F. S. S. Rosa, and D. A. R. Dalvit, Phys. Rev. A 82, 062111 (2010).

[26] R. Messina and M. Antezza, Phys. Rev. A 84, 042102 (2011).

[27] N. Graham, M. Quandt, and H. Weigel, Spectral Methods in Quantum Field Theory, Lecture Notes in Physics (Springer, Berlin, 2009).

[28] I. E. Dzyaloshinkii, E. M. Lifshitz, and L. P. Pitaevskii, Sov. Phys. Usp. 4, 153 (1961).

[29] E. M. Lifschitz and L. P. Pitaevskii, Statistical Physics: Part 2 (Pergamon, Oxford, 1980).

[30] A. Rodriguez, M. Ibanescu, D. Iannuzzi, J. D. Joannopoulos, and S. G. Johnson, Phys. Rev. A 76, 032106 (2007).

[31] S. Pasquali and A. C. Maggs, J. Chem. Phys. 129, 014703 (2008).
[32] J. L. Xiong and W. C. Chew, Appl. Phys. Lett. 95, 154102 (2009).

[33] R. F. Harrington, Field Computation by Moment Methods (Wiley-IEEE, Hoboken, NJ, 1993).

[34] P.-L. George and P. Frey, Mesh Generation (Hermes, London, 2000).

[35] T. H. Boyer, Phys. Rev. A 9, 2078 (1974).

[36] J. Schwinger, L. L. D., Jr., and K. A. Milton, Ann. Phys. 115, 1 (1978).

[37] J. L. Xiong, M. S. Tong, P. Atkins, and W. C. Chew, Phys. Lett. A 374, 2517 (2010).

[38] E. Amooghorban, M. Wubs, N. A. Mortensen, and F. Kheirandish, Phys. Rev. A 84, 013806 (2011).

[39] M. Bordag, D. Robaschik, and E. Wieczorek, Ann. Phys. 165, 192 (1985).

[40] M. E. Peskin and D. V. Schroeder, An Introduction To Quantum Field Theory (Westview Press, Boulder, CO, 1995).

[41] S. Weinberg, The Quantum Theory of Fields (Cambridge University Press, Cambridge, UK, 1995), Vol. 1, Chap. 9.

[42] W. W. Hager, SIAM Rev. 31, 221 (1989).

[43] A careful evaluation of $\gamma^{\mathrm{MM}, \mathrm{r}}$ reveals that the right-hand side of (40) should be augmented by a $\delta$-function term. This $\delta$ function is related to that which enters in the difference between the dyadic Green's functions $\nabla \times \Gamma^{\mathrm{EE}} \times \nabla^{\prime}$ and $\Gamma^{\mathrm{MM}}$; as discussed in Ref. [1], such terms are geometry-independent and make no contribution to physical Casimir quantities, and for this reason we have omitted this term in Eq. (40).

[44] T. Emig, N. Graham, R. L. Jaffe, and M. Kardar, Phys. Rev. Lett. 99, 170403 (2007).

[45] A. Canaguier-Durand, Paulo A. Maia Neto, A. Lambrecht, and S. Reynaud, Phys. Rev. Lett. 104, 040403 (2010).

[46] A. P. McCauley, R. Zhao, M. T. Homer Reid, A. W. Rodriguez, J. Zhou, F. S. S. Rosa, J. D. Joannopoulos, D. A. R. Dalvit, C. M. Soukoulis, and S. G. Johnson, Phys. Rev. B 82, 165108 (2010).

[47] K. A. Milton, P. Parashar, N. Pourtolami, and I. Brevik, Phys. Rev. D 85, 025008 (2012).

[48] C. Eberlein and R. Zietal, Phys. Rev. A 83, 052514 (2011).

[49] A. W. Rodriguez, A. P. McCauley, J. D. Joannopoulos, and S. G. Johnson, Phys. Rev. A 80, 012115 (2009).

[50] A. P. McCauley, A. W. Rodriguez, J. D. Joannopoulos, and S. G. Johnson, Phys. Rev. A 81, 012119 (2010).

[51] M. P. Hertzberg and F. Wilczek, Phys. Rev. Lett. 106, 050404 (2011)

[52] W. C. Chew, J.-M. Jin, C.-C. Lu, E. Michielssen, and J. Song, IEEE Trans. Antennas Propag. 45, 533 (1997).

[53] M. Krüger, T. Emig, G. Bimonte, and M. Kardar, Europhys. Lett. 95, 21002 (2011).

[54] M. Krüger, T. Emig, and M. Kardar, Phys. Rev. Lett. 106, 210404 (2011).

[55] A. W. Rodriguez, O. Ilic, P. Bermel, I. Celanovic, J. D. Joannopoulos, M. Soljačić, and S. G. Johnson, Phys. Rev. Lett. 107, 114302 (2011).

[56] P. Morse and H. Feshbach, Methods of Theoretical Physics (McGraw-Hill, New York, 1953). 
[57] R. Harrington, Time-Harmonic Electromagnetic Fields (IEEE Press, New York, 1961).

[58] P. Waterman, Proc. IEEE 53, 805 (1965).

[59] S. Rengarajan and Y. Rahmat-Samii, Antennas Propag. Mag. IEEE 42, 122 (2000).

[60] A. E. H. Love, Philos. Trans. R. Soc. London A 197, 1 (1901).

[61] S. A. Schelkunoff, Bell Syst. Tech. J. 15, 92 (1936).

[62] J. A. Stratton and L. J. Chu, Phys. Rev. 56, 99 (1939).

[63] L. N. Medgyesi-Mitschang, J. M. Putnam, and M. B. Gedera, J. Opt. Soc. Am. A 11, 1383 (1994).
[64] S. M. Rao, D. R. Wilton, and A. W. Glisson, IEEE Trans. Antennas Propag. 30, 409 (1982).

[65] S. Scheel and S. Y. Buhmann, Acta Phys. Slovaca 58, 675 (2008).

[66] H. Li and M. Kardar, Phys. Rev. Lett. 67, 3275 (1991).

[67] H. Li and M. Kardar, Phys. Rev. A 46, 6490 (1992).

[68] D. Dalvit, P. Milonni, D. Roberts, and F. Rosa, in Casimir Physics, Lecture Notes in Physics Series (Springer, Berlin, 2011).

[69] T. Emig, N. Graham, R. L. Jaffe, and M. Kardar, Phys. Rev. D 77, 025005 (2008). 